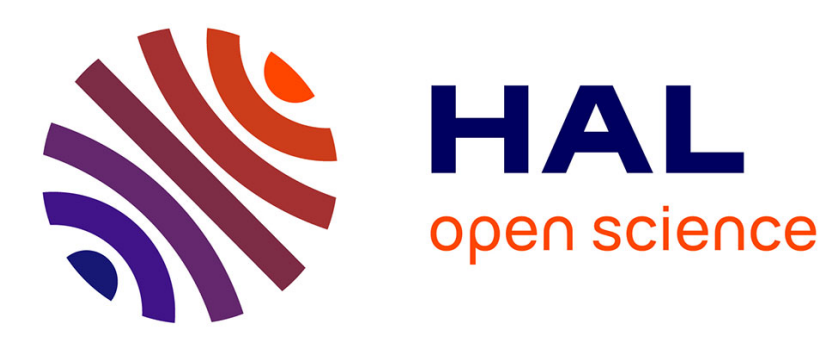

\title{
Variational Analysis and Generalized Equations in Electronics
}

\author{
Samir Adly, Radek Cibulka, Henri Massias
}

\section{To cite this version:}

Samir Adly, Radek Cibulka, Henri Massias. Variational Analysis and Generalized Equations in Electronics. Set-Valued and Variational Analysis, 2013, 21, pp.333-358. 10.1007/s11228-013-0236-5 . hal-00880703

\section{HAL Id: hal-00880703 https://hal.science/hal-00880703}

Submitted on 11 Oct 2018

HAL is a multi-disciplinary open access archive for the deposit and dissemination of scientific research documents, whether they are published or not. The documents may come from teaching and research institutions in France or abroad, or from public or private research centers.
L'archive ouverte pluridisciplinaire HAL, est destinée au dépôt et à la diffusion de documents scientifiques de niveau recherche, publiés ou non, émanant des établissements d'enseignement et de recherche français ou étrangers, des laboratoires publics ou privés. 


\title{
Variational Analysis and Generalized Equations in Electronics

\author{
Stability and Simulation Issues
}

Samir Adly · Radek Cibulka • Henri Massias

\begin{abstract}
The paper is devoted to the study of the Aubin/Lipschitz-like property and the isolated calmness of a particular non-monotone generalized equation arising in electronics. The variational and non-smooth analysis is applied in the theory of non-regular electrical circuits involving electronic devices like ideal diodes, practical diodes, DIACs, silicon controlled rectifiers (SCR), and transistors. We also discuss the relationship of our results to the ones using classical techniques from (smooth) analysis and provide a simulation for several simple electrical circuits which are chosen in order to cover the most common non-smooth elements in electronics. The simulations of the electrical circuits discussed in this paper are performed by using $\mathrm{Xcos}$ (a component of Scilab).
\end{abstract}

Keywords Generalized equation - Non-smooth and variational analysis • Aubin/Lipschitz-like property • Isolated calmness • Non-regular electrical circuits

S. Adly $(\bowtie) \cdot$ R. Cibulka $\cdot$ H. Massias

CNRS-XLIM UMR 7252, Université de Limoges, 123 Avenue Albert Thomas,

87060 Limoges Cedex, France

e-mail: samir.adly@unilim.fr

H. Massias

e-mail: henri.massias@unilim.fr

R. Cibulka

Department of Mathematics, University of West Bohemia, Univerzitní 8,

30614 Pilsen, Czech Republic

e-mail: cibi@kma.zcu.cz 


\section{Introduction}

Given matrices $B \in \mathbb{R}^{n \times m}, C \in \mathbb{R}^{m \times n}$, a non-linear mapping $f: \mathbb{R}^{n} \rightarrow \mathbb{R}^{n}$, and a setvalued map $F: \mathbb{R}^{m} \rightrightarrows \mathbb{R}^{m}$, we consider the problem of finding for a vector $p \in \mathbb{R}^{n}$ the solution $z \in \mathbb{R}^{n}$ to the inclusion

$$
p \in f(z)+B F(C z) .
$$

Denote by $\Phi$ the set-valued mapping from $\mathbb{R}^{n}$ into itself defined by $\Phi(z)=f(z)+$ $B F(C z)$ whenever $z \in \mathbb{R}^{n}$. Our aim is to investigate stability properties such as the Aubin/Lipschitz-like continuity and the isolated calmness of the solution mapping $S:=\Phi^{-1}$ for particular examples arising in electronics. Except for the last section, we will suppose that $m \leq n$, and that

(A1) $B$ is injective;

(A2) $f$ is continuously differentiable on $\mathbb{R}^{n}$;

(A3) $F$ has closed graph;

(A4) $C$ is surjective; and

(A5) there are $F_{j}: \mathbb{R} \rightrightarrows \mathbb{R}, j \in\{1, \ldots, m\}$ such that $F(x)=\prod_{j=1}^{m} F_{j}\left(x_{j}\right)$ whenever $x=$ $\left(x_{1}, \ldots, x_{m}\right)^{T} \in \mathbb{R}^{m}$.

As an example of $F$ satisfying (A3) and (A5), one can mention the Clarke subdifferential of the so-called Moreau-Panagiotopoulos super-potential [5]

$$
J(x):=j_{1}\left(x_{1}\right)+j_{2}\left(x_{2}\right)+\cdots+j_{m}\left(x_{m}\right), \quad x=\left(x_{1}, \ldots, x_{m}\right)^{T} \in \mathbb{R}^{m},
$$

with $j_{i}: \mathbb{R} \rightarrow \mathbb{R}$ being a locally Lipchitz continuous function for each $i=1, \ldots, m$ (corresponding to the electrical super-potential of the electrical devices). This particular case was studied by S. Adly and J. V. Outrata in [1]. They derived criteria for the Aubin/Lipschitz-like continuity and the isolated calmness property of $S$. The general case (1) was investigated in [Adly, S., Cibulka, R.: Quantitative stability of a generalized equation. Application to non-regular electrical circuits. to appear in J. Optim. Theory Appl.] (see Theorems 1 and 3 below). The aim of the present note is twofold. First, to illustrate the application of the theoretical results in case of several important non-smooth elements appearing in electronics (including their inputoutput simulation). Second, to show that one can have $m>n$ in some applications. In such a case, one cannot expect the assumptions (A1) and (A4) to hold. We show, that it is possible to derive the conditions for the isolated calmness property under slightly weaker conditions.

The paper is organized as follows. In the leading section, we provide the needed mathematical background. The next one deals with several examples corresponding to particular non-regular electrical circuits. In the last section, we focus on the isolated calmness of $S$ in case that $m>n$. The assumptions on injectivity of the matrix $B$ and surjectivity of $C$ are weakened.

\section{Mathematical Background}

The notation is fairly standard. In $\mathbb{R}^{d}$, the norm, the scalar product, the closed ball with the center $x \in \mathbb{R}^{d}$ with the radius $r \geq 0$, and the unit ball are denoted by $\|\cdot\|$, $\langle\cdot, \cdot\rangle, \mathbb{B}(x, r)$, and $\mathbb{B}$, respectively. 
Given a non-empty subset $\Omega$ of $\mathbb{R}^{d}$, the regular (Fréchet) normal cone to $\Omega$ at $\bar{x} \in \Omega$ is the set

$$
\widehat{N}(\bar{x} ; \Omega):=\left\{\xi \in \mathbb{R}^{d}: \limsup _{\Omega \ni x \rightarrow \bar{x}} \frac{\langle\xi, x-\bar{x}\rangle}{\|x-\bar{x}\|} \leq 0\right\} ;
$$

the Mordukhovich limiting normal cone $N(\bar{x} ; \Omega)$ to $\Omega$ at $\bar{x}$ contains all $\xi \in \mathbb{R}^{d}$ for which there are sequences $\left(x^{k}\right)_{k \in \mathbb{N}}$ in $\Omega$ and $\left(\xi^{k}\right)_{k \in \mathbb{N}}$ in $\mathbb{R}^{d}$ converging to $\bar{x}$ and $\xi$, respectively, such that

$$
\xi^{k} \in \widehat{N}\left(x^{k} ; \Omega\right) \quad \text { for each } k \in \mathbb{N}
$$

and finally the Bouligand-Severi tangent cone $T(\bar{x} ; \Omega)$ to $\Omega$ at $\bar{x}$ contains those $v \in \mathbb{R}^{d}$ for which there are sequences $\left(t^{k}\right)_{k \in \mathbb{N}}$ in $(0, \infty)$ and $\left(v^{k}\right)_{k \in \mathbb{N}}$ in $\mathbb{R}^{d}$ converging to 0 and $v$, respectively, such that $\bar{x}+t^{k} v^{k} \in \Omega$ whenever $k \in \mathbb{N}$. The set $\Omega$ is called normally (Clarke) regular at $\bar{x}$ if $\widehat{N}(\bar{x} ; \Omega)=N(\bar{x} ; \Omega)$.

By $S: \mathbb{R}^{d} \rightrightarrows \mathbb{R}^{l}$ we mean a multivalued mapping from $\mathbb{R}^{d}$ into $\mathbb{R}^{l}$ with the domain $\operatorname{dom} S$, the graph gph $S$ and the range $\operatorname{rge} S$. Fix a point $(\bar{x}, \bar{y}) \in \operatorname{gph} S$, a mapping $S$ is said to have the Aubin/Lipschitz-like property around $(\bar{x}, \bar{y})$ if there is a constant $\kappa \geq 0$ along with neighborhoods $U$ of $\bar{x}$ and $V$ of $\bar{y}$ such that

$$
S(x) \cap V \subset S\left(x^{\prime}\right)+\kappa\left\|x-x^{\prime}\right\| \mathbb{B} \quad \text { for all } \quad x, x^{\prime} \in U .
$$

The infimum of the set of values $\kappa$ for which there are neighborhoods $U$ of $\bar{x}$ and $V$ of $\bar{y}$ such that (2) holds is called Lipschitz modulus of $S$ at $(\bar{x}, \bar{y})$ and is denoted by $\operatorname{lip}(S ;(\bar{x}, \bar{y}))$. It is well known [2, Theorem 3E.6] that the Aubin/Lipschitz-like property of $S$ around $(\bar{x}, \bar{y})$ is equivalent to the metric regularity of $\Phi:=S^{-1}$ around $(\bar{y}, \bar{x})$ and that the regularity modulus of $\Phi$ at $(\bar{y}, \bar{x})$ equals to the Lipschitz modulus of $S$ at $(\bar{x}, \bar{y})$. Clearly, this property ensures that $S(x) \neq \emptyset$ for each $x$ in vicinity of $\bar{x}$.

Fixing one of the points in $U$, i.e. the request for the existence of a constant $\kappa \geq 0$ along with neighborhoods $U$ of $\bar{x}$ and $V$ of $\bar{y}$ such that

$$
S(x) \cap V \subset S(\bar{x})+\kappa\|x-\bar{x}\| \mathbb{B} \quad \text { whenever } \quad x \in U,
$$

we get the definition of the calmness property of $S$ at $(\bar{x}, \bar{y})$. Again, the infimum of the set of values $\kappa$ for which there are neighborhoods $U$ of $\bar{x}$ and $V$ of $\bar{y}$ such that (3) holds is called calmness modulus of $S$ at $(\bar{x}, \bar{y})$ and is denoted by $\operatorname{clm}(S ;(\bar{x}, \bar{y}))$. Remember, that [2, Theorem 3H.3] establishes the equivalence between calmness property of $S$ at $(\bar{x}, \bar{y})$ and the metric subregularity of $\Phi$ at $(\bar{y}, \bar{x})$. Moreover, the subregularity modulus of $\Phi$ at $(\bar{y}, \bar{x})$ and the calmness modulus of $S$ at $(\bar{x}, \bar{y})$ are the same. However, there is no guarantee that $S(x) \neq \emptyset$ for any $x$ in vicinity of $\bar{x}$.

Further, the mapping $S$ has the isolated calmness property at $(\bar{x}, \bar{y})$ provided that it has calmness property and $\bar{y}$ is an isolated point of $S(\bar{x})$, i.e. $S(\bar{x}) \cap \mathbb{B}(\bar{y}, r)=\{\bar{y}\}$ for some $r>0$. Note that the isolated calmness property of $S$ at $(\bar{x}, \bar{y})$ amounts to the existence of a constant $\kappa \geq 0$ along with neighborhoods $U$ of $\bar{x}$ and $V$ of $\bar{y}$ such that

$$
S(x) \cap V \subset \bar{y}+\kappa\|x-\bar{x}\| \mathbb{B} \quad \text { for each } \quad x \in U .
$$

Theorem 3I.2 in [2] says that the isolated calmness property of $S$ at $(\bar{x}, \bar{y})$ is equivalent to the strong metric subregularity of $\Phi$ at $(\bar{y}, \bar{x})$. Again, one can have $S(x)=\emptyset$ for all $x$ in a neighborhood of $\bar{x}$. This is not the case if the corresponding calmness modulus is positive. 
For now on assume that $\Phi$ and $S$ are the mappings defined in the introduction. For a point $(\bar{z}, \bar{p}) \in \operatorname{gph} \Phi$, we set $\bar{v}:=\left(B^{T} B\right)^{-1} B^{T}(\bar{p}-f(\bar{z}))$. In [Adly, S., Cibulka, R.: Quantitative stability of a generalized equation. Application to non-regular electrical circuits. to appear in J. Optim. Theory Appl.], using the well-known Mordukhovich criterion [4, Theorem 4.10], the following necessary and sufficient condition for the Aubin/Lipschitz-like continuity of $S:=\Phi^{-1}$ is derived.

Theorem 1 Suppose that the assumptions $(A 1)-(A 4)$ hold true. Then $S$ has the Aubin/Lipschitz-like property at $(\bar{p}, \bar{z}) \in \mathrm{gph} S$ if and only if

$$
\left.\begin{array}{c}
\left(\left(C C^{T}\right)^{-1} C \nabla f(\bar{z})^{T} \xi, B^{T} \xi\right) \in-N((C \bar{z}, \bar{v}) ; \operatorname{gph} F) \\
\nabla f(\bar{z})^{T} \xi \in \operatorname{rge} C^{T}
\end{array}\right\} \Longrightarrow \xi=0_{\mathbb{R}^{n}}
$$

Using [2, Theorem 4C.1], one can obtain a similar result on the isolated calmness property of $S$.

Theorem 2 Suppose that the assumptions $(A 1)-(A 4)$ hold true. Then $S$ has the isolated calmness property at $(\bar{p}, \bar{z}) \in \mathrm{gph} S$ if and only if

$$
\left.\begin{array}{c}
\left(C b,-\left(B^{T} B\right)^{-1} B^{T} \nabla f(\bar{z}) b\right) \in T((C \bar{z}, \bar{v}) ; \operatorname{gph} F) \\
\nabla f(\bar{z}) b \in \operatorname{rge} B
\end{array}\right\} \Longrightarrow b=0 \mathbb{R}_{\mathbb{R}^{n}}
$$

Remark 1 In view of [6, Proposition 6.41], the assumption (A5) ensures that the conditions (5) and (6) can be checked coordinate-wise. To be more precise, as $\operatorname{gph} F=\prod_{j=1}^{m} \operatorname{gph} F_{j}$, one has $N((C \bar{z}, \bar{v})$; gph $F)=\prod_{j=1}^{m} N\left(\left((C \bar{z})_{j}, \bar{v}_{j}\right)\right.$; gph $\left.F_{j}\right)$ and $T((C \bar{z}, \bar{v}) ; \operatorname{gph} F) \subset \prod_{j=1}^{m} T\left(\left((C \bar{z})_{j}, \bar{v}_{j}\right)\right.$; gph $\left.F_{j}\right)$. Hence, replacing the tangent cone to the graph of $F$ by the product of tangent cones to the graphs of $F_{j}$ in (6), one gets the sufficient condition for the isolated calmness. It becomes necessary if, in addition, gph $F_{j}$ is normally regular at $\left((C \bar{z})_{j}, \bar{v}_{j}\right)$ for each $j \in\{1, \ldots, m\}$.

Recall that $M \in \mathbb{R}^{n \times n}$ is called a $P$-matrix provided that all its $k$-by- $k$ principal minors are positive whenever $k \in\{1, \ldots, n\}$. It is well known, that $M$ is a P-matrix if and only if for any non-zero $x \in \mathbb{R}^{n}$ there is $j \in\{1, \ldots, n\}$ such that $x_{j}(M x)_{j}>0$. The following simple sufficient condition for the Aubin/Lipschitz-like property may be useful.

Corollary 1 In addition to $(A 1)-(A 5)$, assume that $n=m$, that $B=C=I_{n}$, that $\nabla f(\bar{z})^{T}$ is a $P$-matrix, and that for each $j \in\{1,2, \ldots, n\}$, we have

$$
N\left(\left(\bar{z}_{j}, \bar{v}_{j}\right) ; \operatorname{gph} F_{j}\right) \subset\left\{(a, b)^{T} \in \mathbb{R}^{2}: a b \leq 0\right\} .
$$

Then $S$ has the Aubin/Lipschitz-like property at $(\bar{p}, \bar{z}) \in \operatorname{gph} S$.

Proof In view of Remark 1, the condition (5) says that $S$ has the Aubin/Lipschitz-like property at $(\bar{p}, \bar{z})$ provided that $\xi=0_{\mathbb{R}^{n}}$ is the only point which satisfies

$$
\left(\nabla f(\bar{z})^{T} \xi, \xi\right) \in-\prod_{j=1}^{n} N\left(\left(\bar{z}_{j}, \bar{v}_{j}\right) ; \operatorname{gph} F_{j}\right) \subset\left[\left\{(a, b)^{T} \in \mathbb{R}^{2}: a b \leq 0\right\}\right]^{n} .
$$


Suppose on the contrary that $\xi$ is non-zero. Then $\xi_{j}\left(\nabla f(\bar{z})^{T} \xi\right)_{j} \leq 0$ for each $j \in$ $\{1,2, \ldots, n\}$. We obtained a contradiction, since $\nabla f(\bar{z})^{T}$ is a P-matrix.

Note that the inclusion in Corollary 1 is satisfied if $F_{j}$ is a maximal monotone operator. We recall that an operator $H: \mathbb{R} \rightrightarrows \mathbb{R}$ is maximal monotone if and only if there is a proper lower semi-continuous convex function $\psi: \mathbb{R} \rightarrow \mathbb{R} \cup\{+\infty\}$ such that $H=\partial \psi$, where the subdifferential is understood in the sense of convex analysis.

\section{Non-Regular Electrical Circuits}

In this section we investigate the stability properties of the solution mappings corresponding to several non-regular electrical circuits.

Example 1 Consider the circuit in Fig. 1 involving a load resistance $R>0$, a source $E>0$, an input-signal source $u$ with corresponding instantaneous current $i$, and a non-smooth element with the current-voltage (i-v) characteristic given by a setvalued mapping $F: \mathbb{R} \rightrightarrows \mathbb{R}$. Kirchhoff's voltage law reveals that

$$
u-E \in \underbrace{R i}_{V_{R}}+\underbrace{F(i)}_{V_{D}} .
$$

By setting $p=u-E$ and $z=i$ we get (1) with $m=n=1$, that $B=C=1$, and $f(z)=R z, z \in \mathbb{R}$.

Practical Diode Suppose that $V_{1}<0<V_{2}$. The $\mathrm{i}-\mathrm{v}$ characteristic is given by

$$
F(z):= \begin{cases}{\left[V_{1}, V_{2}\right],} & \text { if } z=0 \\ V_{2}, & \text { if } z>0 \\ V_{1}, & \text { if } z<0\end{cases}
$$

Fig. 1 The circuit considered in Example 1

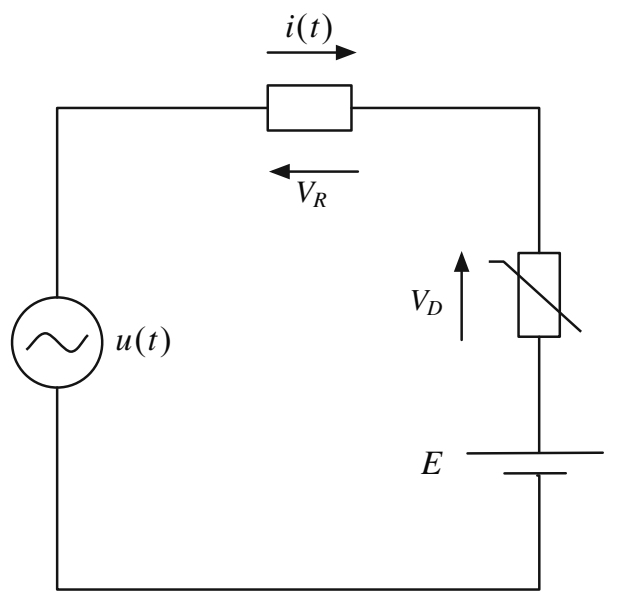


Note that for each $p \in \mathbb{R}$ there is a unique solution $z \in \mathbb{R}$ to (1) given by

$$
z= \begin{cases}0, & \text { if } \quad p \in\left[V_{1}, V_{2}\right], \\ \left(p-V_{2}\right) / R, & \text { if } p>0 \\ \left(p-V_{1}\right) / R, & \text { if } \quad p<0 .\end{cases}
$$

Since $F$ is maximal monotone, one applies the Corollary 1 to conclude that $S$ has the Aubin/Lipschitz-like property at any $(\bar{p}, \bar{z}) \in \operatorname{gph} S$. Moreover, the uniqueness of the solution implies that $S$ has also the isolated calmness property at any reference point. In order to simulate this circuit in standard software packages, one has to replace the multivalued map $F$ by its single-valued "good" approximation, $\varphi$ say. For example, for a given small $\varepsilon>0$ one can take

$$
\varphi(z)= \begin{cases}\frac{1}{\varepsilon}\left(V_{2}-\frac{V_{1}+V_{2}}{2}\right) z+\frac{V_{1}+V_{2}}{2}, & \text { if } z \in[-\varepsilon, \varepsilon], \\ V_{2}, & \text { if } z>\varepsilon, \\ V_{1}, & \text { if } z<-\varepsilon .\end{cases}
$$

For each $p \in \mathbb{R}$, the (unique) solution to the equation $p=f(z)+\varphi(z)$ is close to the solution of (1). Moreover, since the monotonicity is preserved, the corresponding solution mapping also has both the Aubin/Lipschitz-like and the isolated calmness property around any reference point of its graph. The simulation can be seen in Fig. 2.
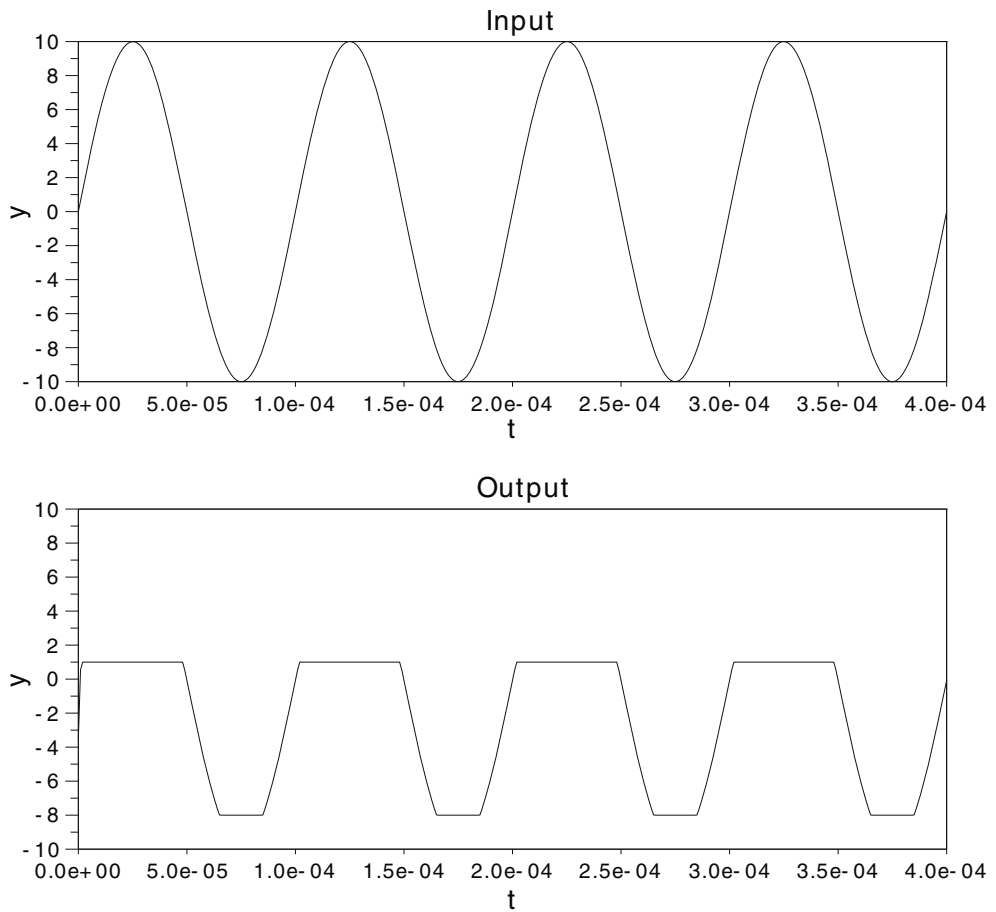

Fig. 2 Voltage of the input source and the output voltage across the practical diode $\left(R=10, V_{1}=\right.$ $-8, V_{2}=1, \varepsilon=0.01$ ) 
Diode Alternating Current (DIAC) Bilateral triggers diacs are bidirectional thyristors designed to switch alternating current and trigger silicon controlled rectifiers and triacs. Figure 3 illustrates typical $\mathrm{i}-\mathrm{v}$ characteristic of a diac along with its schematic symbol. Here $V$ (resp. $-V$ ) is the forward (resp. reverse) breakover voltage. The diacs, because of their symmetrical bidirectional switching characteristics, are widely used as triggering devices in triac phase control circuits employed for lamp dimmer, heat control, universal motor speed control etc.

Suppose that $V>0$ and $a>0$ are given. The $\mathrm{i}-\mathrm{v}$ characteristic (see Fig. 3) is given by

$$
F(z):= \begin{cases}{[-V, V],} & \text { if } z=0 \\ V / \sqrt{2 a z / V+1,} & \text { if } z>0 \\ -V / \sqrt{-2 a z / V+1}, & \text { if } z<0\end{cases}
$$

Note that for each $p \in \mathbb{R}$ there is a solution $z \in \mathbb{R}$ to the equation (1). Indeed, as $\Phi(z)=R z+F(z), z \in \mathbb{R}$, we have $\operatorname{rge} \Phi=\mathbb{R}$. Moreover, this solution is unique if $a \leq R$, since for a non-zero $z \in \mathbb{R}$ one infers that

$$
\Phi^{\prime}(z)=R-\frac{a}{\left(\frac{2 a|z|}{V}+1\right)^{3 / 2}}=\frac{R\left(\frac{2 a|z|}{V}+1\right)^{3 / 2}-a}{\left(\frac{2 a|z|}{V}+1\right)^{3 / 2}}>\frac{R-a}{\left(\frac{2 a|z|}{V}+1\right)^{3 / 2}} \geq 0 .
$$

In this case, the solution equals to zero if $p \in[-V, V]$, it is positive if $p>V$ and negative when $p<V$.

Clearly, we can split $\Phi$ into different functions $f$ and $F$ which produce the same inclusion (1). Namely, from now on, we assume that

$$
f(z)=\left\{\begin{array}{ll}
R z+\frac{V}{\sqrt{2 a z / V+1}-V,} \quad z \geq 0, \\
R z-\frac{V}{\sqrt{-2 a z / V+1}}+V, & z<0,
\end{array} \quad \text { and } F(z):= \begin{cases}{[-V, V],} & z=0, \\
V, & z>0, \\
-V, & z<0 .\end{cases}\right.
$$

So,

$$
f^{\prime}(z)=R-\frac{a}{\left(\frac{2 a|z|}{V}+1\right)^{3 / 2}} \quad \text { whenever } \quad z \in \mathbb{R}
$$

Fig. 3 The DIAC schematic symbol and its $\mathrm{i}-\mathrm{V}$ characteristic

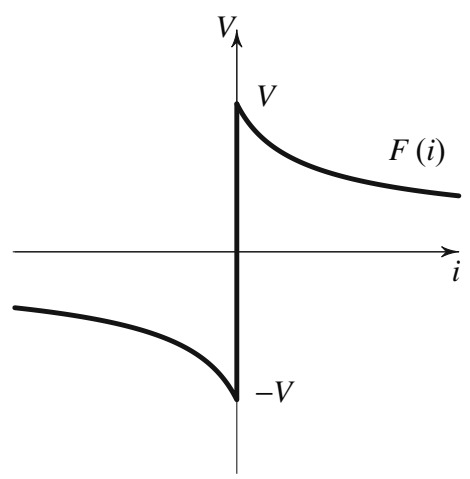


Indeed, $f^{\prime}(z)=\Phi^{\prime}(z)$ if $z \neq 0$ and $f^{\prime}(0)=R-a$ as can be easily justified, e.g.,

$$
\lim _{h \rightarrow 0+} \frac{R h+\frac{V}{\sqrt{\frac{2 a h}{V}+1}}-V}{h}=\lim _{h \rightarrow 0+}\left(R-\frac{a}{\left(\frac{2 a h}{V}+1\right)^{\frac{3}{2}}}\right)=R-a .
$$

For a fixed $\bar{p} \in \mathbb{R}$, let $\bar{z}$ be the corresponding solution to (1). Now, (5) reads as

$$
\left(f^{\prime}(\bar{z}) \xi, \xi\right) \in-N((\bar{z}, \bar{p}-f(\bar{z})) ; \operatorname{gph} F) \Longrightarrow \xi=0
$$

where $N((x, y)$; gph $F)$ is given by

$$
\begin{cases}\mathbb{R}\left(\begin{array}{l}
0 \\
1
\end{array}\right), & \text { if } x>0, y=V \text { or } x<0, y=-V, \\
\mathbb{R}\left(\begin{array}{l}
1 \\
0
\end{array}\right), & \text { if } x=0, y \in(-V, V), \\
\mathbb{R}\left(\begin{array}{l}
0 \\
1
\end{array}\right) \cup \mathbb{R}\left(\begin{array}{l}
1 \\
0
\end{array}\right) \cup \operatorname{cone}\left\{\left(\begin{array}{l}
0 \\
1
\end{array}\right),\left(\begin{array}{c}
-1 \\
0
\end{array}\right)\right\}, & \text { if } x=0, y=V, \\
\mathbb{R}\left(\begin{array}{l}
0 \\
1
\end{array}\right) \cup \mathbb{R}\left(\begin{array}{l}
1 \\
0
\end{array}\right) \cup \text { cone }\left\{\left(\begin{array}{c}
0 \\
-1
\end{array}\right),\left(\begin{array}{l}
1 \\
0
\end{array}\right)\right\}, & \text { if } x=0, y=-V, \\
\varnothing, & \text { otherwise. }\end{cases}
$$

If $a<R$, then $f^{\prime}(z)>0$ for each $z \in \mathbb{R}$. So, $S$ has the Aubin/Lipschitz-like property at $(\bar{p}, \bar{z})$. Again, the uniqueness of the solution implies that $S$ has also the isolated calmness property at this point.

If $a=R$, then $f^{\prime}(z)>0$ for each non-zero $z \in \mathbb{R}$ and $f^{\prime}(0)=0$. Hence $S$ has both the Aubin/Lipschitz-like and the isolated calmness property at $(\bar{p}, \bar{z})$ provided that $\bar{p} \notin\{-V, V\}$. If $\bar{p} \in\{-V, V\}$ then $S$ has not the Aubin/Lipschitz-like property at $(\bar{p}, \bar{z})$.

The condition (6) has the form

$$
\left(b,-f^{\prime}(\bar{z}) b\right) \in T((\bar{z}, \bar{p}-f(\bar{z})) ; \operatorname{gph} F) \Longrightarrow b=0,
$$

with

$$
T((x, y) ; \operatorname{gph} F)= \begin{cases}\mathbb{R}\left(\begin{array}{l}
1 \\
0
\end{array}\right), & \text { if } x>0, y=V \text { or } x<0, y=-V, \\
\mathbb{R}\left(\begin{array}{l}
0 \\
1
\end{array}\right), & \text { if } x=0, y \in(-V, V), \\
\mathbb{R}_{+}\left(\begin{array}{l}
1 \\
0
\end{array}\right) \cup \mathbb{R}_{+}\left(\begin{array}{c}
0 \\
-1
\end{array}\right), & \text { if } x=0, y=V, \\
\mathbb{R}_{+}\left(\begin{array}{c}
-1 \\
0
\end{array}\right) \cup \mathbb{R}_{+}\left(\begin{array}{l}
0 \\
1
\end{array}\right), & \text { if } x=0, y=-V, \\
\emptyset & \text { otherwise. }\end{cases}
$$


Therefore, if $a=R$, the mapping $S$ has not even the isolated calmness property at the reference point if $\bar{p} \in\{-V, V\}$.

The case $a>R$ could be discussed similarly, but the solution is no more unique. For example, if $\bar{z}=0$ then $f^{\prime}(0)<0$ and $f(0)=0$. Thus $S$ has both the Aubin/Lipschitz-like and the isolated calmness property at $(\bar{p}, \bar{z})$ if $\bar{p} \in(-V, V)$. If $\bar{p} \in\{-V, V\}$ then $S$ has the isolated calmness property at the reference point but not the Aubin/Lipschitz-like property. Let us summarize the above consideration in Table 1.

Let us replace the $\mathrm{i}-\mathrm{v}$ characteristic of the DIAC by its single-valued approximation $\varphi$ defined by

$$
\varphi(z)= \begin{cases}\frac{V}{\varepsilon \sqrt{2 a \varepsilon / V+1}} z, & \text { if } z \in[-\varepsilon, \varepsilon] \\ V / \sqrt{2 a z / V+1}, & \text { if } z>\varepsilon \\ -V / \sqrt{-2 a z / V+1}, & \text { if } z<-\varepsilon\end{cases}
$$

with $\varepsilon>0$ sufficiently small. Therefore,

$$
\varphi^{\prime}(z)=\left\{\begin{array}{lll}
\alpha:=\frac{V}{\varepsilon \sqrt{2 a \varepsilon} / V+1}, & \text { if } & |z|<\varepsilon, \\
\beta_{z}:=-a(2 a|z| / V+1)^{-3 / 2}, & \text { if } & |z|>\varepsilon .
\end{array}\right.
$$

Put $\widetilde{\Phi}(z)=R z+\varphi(z), z \in \mathbb{R}$. As rge $\widetilde{\Phi}=\mathbb{R}$, there is a solution to the equation $p=$ $\widetilde{\Phi}(z)$ for each $p \in \mathbb{R}$. If $a>0$ is such that $a \leq R(2 a \varepsilon / V+1)^{3 / 2}$, then $\widetilde{\Phi}^{\prime}(z)>0$ for any $z \in \mathbb{R}$ with $|z| \neq \varepsilon$. Hence this assumption guarantees the uniqueness. Clearly, any solution to the equation is close to the solution of (1).

Let $\bar{z} \in \mathbb{R}$. Now, (5) reads as

$$
(R \xi, \xi) \in-N((\bar{z}, \varphi(\bar{z})) ; \operatorname{gph} \varphi) \Longrightarrow \xi=0,
$$

where $N((z, \varphi(z)) ; \operatorname{gph} \varphi)$ equals to

$$
\begin{cases}\mathbb{R}\left(\begin{array}{c}
-\alpha \\
1
\end{array}\right), & \text { if }|z|<\varepsilon, \\
\mathbb{R}\left(\begin{array}{c}
-\beta_{z} \\
1
\end{array}\right), & \text { if }|z|>\varepsilon, \\
\mathbb{R}_{+}\left(\begin{array}{c}
-\alpha \\
1
\end{array}\right) \cup \mathbb{R}_{+}\left(\begin{array}{c}
-\beta_{z} \\
1
\end{array}\right) \cup \operatorname{cone}\left\{\left(\begin{array}{c}
-\alpha \\
1
\end{array}\right),\left(\begin{array}{c}
-\beta_{z} \\
1
\end{array}\right)\right\}, & \text { if } z=\varepsilon, \\
\mathbb{R}_{+}\left(\begin{array}{c}
\alpha \\
-1
\end{array}\right) \cup \mathbb{R}_{+}\left(\begin{array}{c}
\beta_{z} \\
-1
\end{array}\right) \cup \operatorname{cone}\left\{\left(\begin{array}{c}
\alpha \\
-1
\end{array}\right),\left(\begin{array}{c}
\beta_{z} \\
-1
\end{array}\right)\right\}, & \text { if } z=-\varepsilon .\end{cases}
$$

Table 1 Properties of the solution mapping for DIAC

\begin{tabular}{lllll}
\hline & $(\bar{p}, \bar{z}) \in(-V, V) \times\{0\}$ & $(\bar{p}, \bar{z}) \in\{-V, V\} \times\{0\}$ & $\bar{p}>V$ & $\bar{p}<-V$ \\
\hline$a<R$ & AP and IC & AP and IC & AP and IC & AP and IC \\
$a=R$ & AP and IC & None & AP and IC & AP and IC \\
$a>R$ & AP and IC & IC & Not discussed $^{\mathrm{a}}$ & Not discussed
\end{tabular}

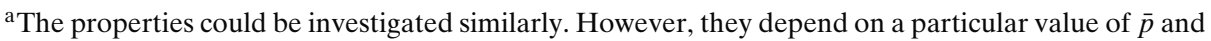
the corresponding solution $\bar{z}$ 
The condition (6) has the form

$$
(b,-R b) \in T((\bar{z}, \varphi(\bar{z})) ; \operatorname{gph} \varphi) \Longrightarrow b=0,
$$

with

$$
T((z, \varphi(z)) ; \operatorname{gph} \varphi)= \begin{cases}\mathbb{R}\left(\begin{array}{l}
1 \\
\alpha
\end{array}\right), & \text { if }|z|<\varepsilon, \\
\mathbb{R}\left(\begin{array}{c}
1 \\
\beta_{z}
\end{array}\right), & \text { if }|z|>\varepsilon, \\
\mathbb{R}_{+}\left(\begin{array}{l}
-1 \\
-\alpha
\end{array}\right) \cup \mathbb{R}_{+}\left(\begin{array}{c}
1 \\
\beta_{z}
\end{array}\right), & \text { if } z=\varepsilon, \\
\mathbb{R}_{+}\left(\begin{array}{l}
1 \\
\alpha
\end{array}\right) \cup \mathbb{R}_{+}\left(\begin{array}{c}
-1 \\
-\beta_{z}
\end{array}\right), & \text { if } z=-\varepsilon .\end{cases}
$$

Using similar steps as in the case of $S$ one can establish the stability properties of $\widetilde{S}:=(\widetilde{\Phi})^{-1}$ (see the Table 2 ).

Therefore, we can expect the same qualitative behaviour as in the case of the original solution mapping. However, one has to adjust the value of the parameters involved in the model. The simulation can be seen in Fig. 4.

Silicon Controlled Rectifier (SCR) Silicon controlled rectifiers are three-terminal devices designed for start/stop control circuit for a direct current motor, lamp or other practical load. The SCR is used in many applications: motor controls, timedelay circuits, heater controls, phase controls, relay controls to name a few.

Suppose that $V_{1}<0, \alpha>0$ and $a>0$ are given numbers, and that $\varphi: \mathbb{R} \rightarrow(0, \infty)$ is a continuously differentiable function with $\varphi(\alpha)<\varphi(0), \varphi^{\prime}(0)>0$ and $\varphi^{\prime}(\alpha)>0$. We suppose that the $\mathrm{i}-\mathrm{v}$ characteristic (see Fig. 5) is given by

$$
F(z):= \begin{cases}{\left[V_{1}, \varphi(0)\right],} & \text { if } z=0 \\ a z+V_{1}, & \text { if } z<0 \\ \varphi(z), & \text { if } z \in[0, \alpha] \\ a(z-\alpha)+\varphi(\alpha), & \text { if } z>\alpha\end{cases}
$$

Table 2 Properties of the solution mapping for the approximation of DIAC

\begin{tabular}{llll}
\hline & $|\bar{z}|<\varepsilon$ & $|\bar{z}|=\varepsilon$ & $|\bar{z}|>\varepsilon$ \\
\hline$a<R(2 a \varepsilon / V+1)^{3 / 2}$ & AP and IC & AP and IC & AP and IC \\
$a=R(2 a \varepsilon / V+1)^{3 / 2}$ & AP and IC & None & AP and IC \\
$a>R(2 a \varepsilon / V+1)^{3 / 2}$ & AP and IC & IC & Not discussed $^{\text {a }}$ \\
\hline
\end{tabular}

a The properties could be investigated similarly. However, they depend on a particular value of $\bar{p}$ and the corresponding solution $\bar{z}$ 

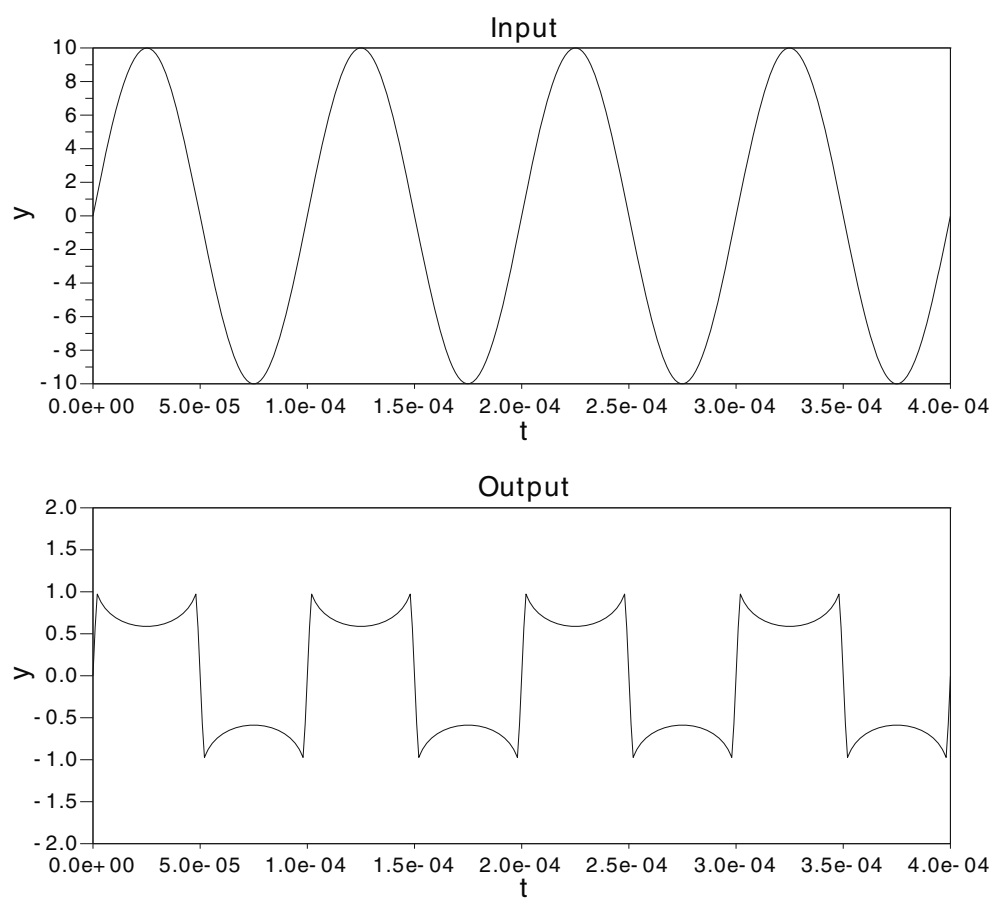

Fig. 4 Voltage of the input source and the output voltage across the DIAC $(R=10, V=2, a=10$, $\varepsilon=0.01)$

Note that for each $p \in \mathbb{R}$ there is a solution (not necessarily unique) $z \in \mathbb{R}$ to (1). Fix any $(\bar{p}, \bar{z}) \in \operatorname{gph} S$. Now, (5) and (6) read as

$$
(R \xi, \xi) \in-N((\bar{z}, \bar{p}-R \bar{z}) ; \operatorname{gph} F) \Longrightarrow \xi=0,
$$

and

$$
(b,-R b) \in T((\bar{z}, \bar{p}-R \bar{z}) ; \operatorname{gph} F) \Longrightarrow b=0,
$$

Fig. 5 The Silicon controlled rectifier schematic symbol and its typical $\mathrm{i}-\mathrm{v}$ characteristics

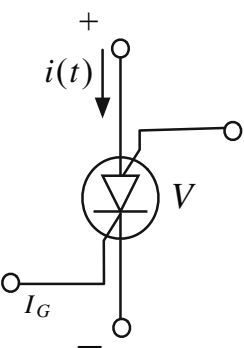

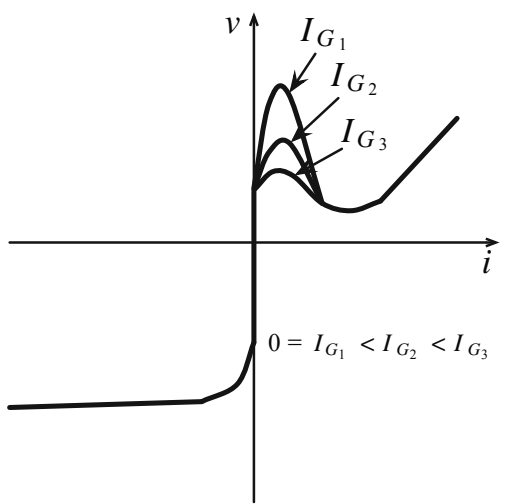


respectively, where $N((x, y) ; \operatorname{gph} F)$ equals to

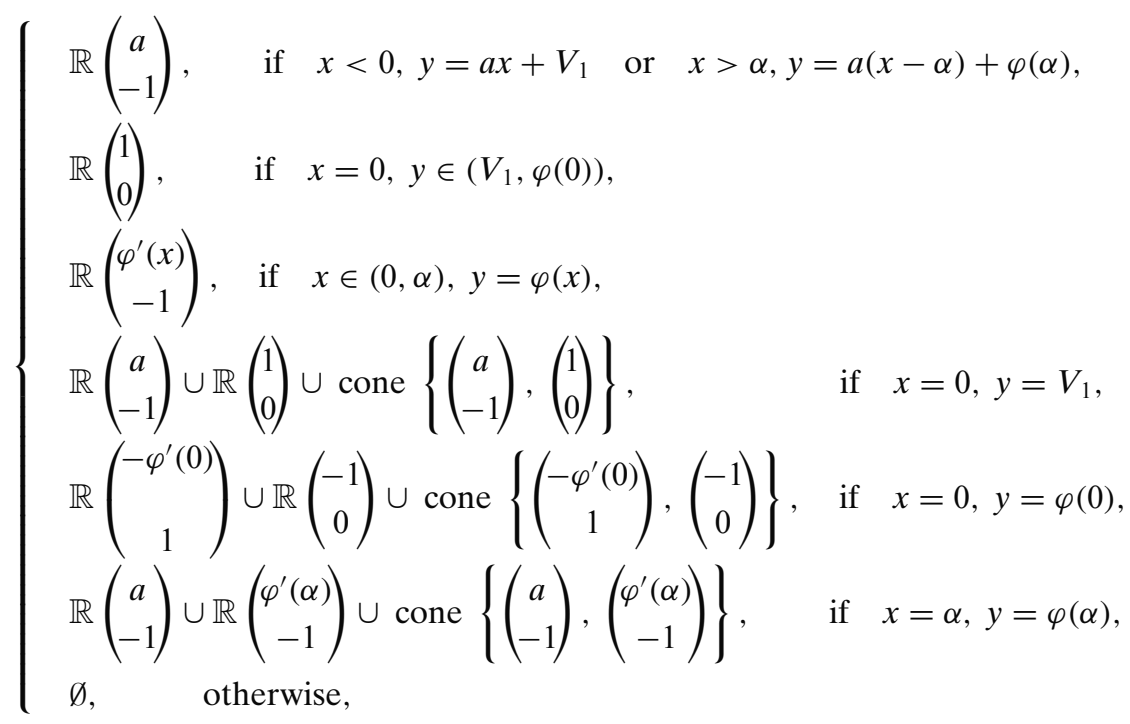

and $T((x, y) ; \operatorname{gph} F)$ equals to

$$
\begin{cases}\mathbb{R}\left(\begin{array}{l}
1 \\
a
\end{array}\right), \quad \text { if } \quad x<0, y=a x+V_{1} & \text { or } \quad x>\alpha, y=a(x-\alpha)+\varphi(\alpha), \\
\mathbb{R}\left(\begin{array}{l}
0 \\
1
\end{array}\right), & \text { if } x=0, y \in\left(V_{1}, \varphi(0)\right), \\
\mathbb{R}\left(\begin{array}{c}
1 \\
\varphi^{\prime}(x)
\end{array}\right), & \text { if } x \in(0, \alpha), y=\varphi(x), \\
\mathbb{R}_{+}\left(\begin{array}{c}
-1 \\
-a
\end{array}\right) \cup \mathbb{R}_{+}\left(\begin{array}{l}
0 \\
1
\end{array}\right), & \text { if } x=0, y=V_{1}, \\
\mathbb{R}_{+}\left(\begin{array}{c}
1 \\
\varphi^{\prime}(0)
\end{array}\right) \cup \mathbb{R}_{+}\left(\begin{array}{c}
0 \\
-1
\end{array}\right), & \text { if } x=0, y=\varphi(0), \\
\mathbb{R}_{+}\left(\begin{array}{c}
-1 \\
-\varphi^{\prime}(\alpha)
\end{array}\right) \cup \mathbb{R}_{+}\left(\begin{array}{c}
1 \\
a
\end{array}\right), & \text { if } x=\alpha, y=\varphi(\alpha), \\
\emptyset, & \text { otherwise. }\end{cases}
$$

Therefore, $S$ has both the Aubin/Lipschitz-like and the isolated calmness property for each $(\bar{p}, \bar{z}) \in \operatorname{gph} S$ with $\bar{z} \notin(0, \alpha)$. On the other hand, if $\bar{z} \in(0, \alpha)$ then $S$ has these properties if and only if $\varphi^{\prime}(\bar{z}) \neq-R$. Again, a single-valued approximation of $F$ as in the previous cases will not effect the qualitative behaviour of the solution map. The simulation can be seen in Fig. 6 .

Example 2 (SCR and Zener Diode in parallel) Consider the circuit in Fig. 7 involving a load resistance $R>0$, two sources $E_{2}>E_{1}>0$, an input-signal source $u$ with 

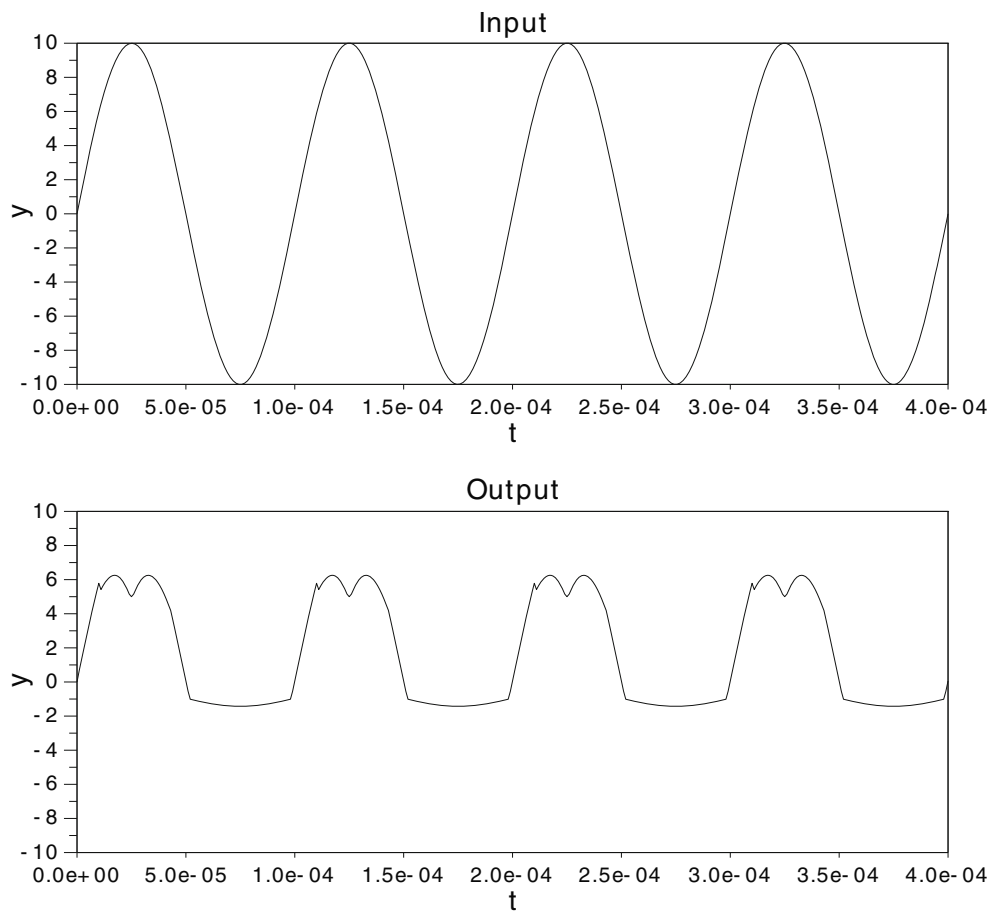

Fig. 6 Voltage of the input source and the output voltage across the SCR $\left(R=10, V_{1}=1, a=0.5\right.$, $\left.\alpha=1.3, \varphi(z)=24 z^{3}-46 z^{2}+19 z+4, \varepsilon=0.01\right)$

corresponding instantaneous current $i$, and two non-smooth elements - the SCR and the Zener Diode. A Zener diode is made to permit current to flow in the reverse direction if the voltage is larger than the rated breakdown. The schematic symbol and the $\mathrm{i}-\mathrm{v}$ characteristic of a diode are depicted in Fig. 8. The Zener diode is a good voltage regulator to maintain a constant voltage regardless of minor variations in load current or input voltage.

Fig. 7 The circuit considered in Example 2

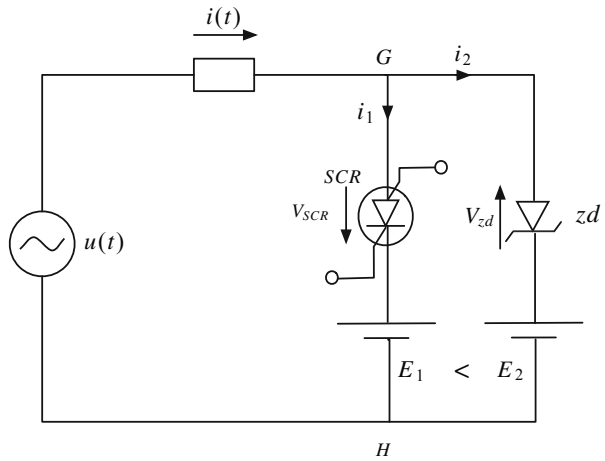



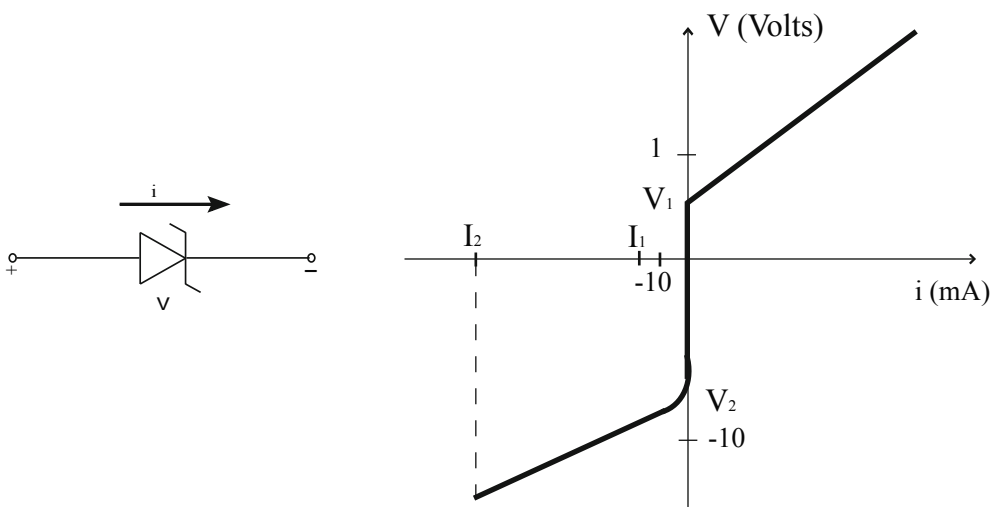

Fig. 8 The Zener diode schematic symbol and its typical i-v characteristic (we assume $V_{1}=-V_{2}=V$ )

Suppose that $V>0, V_{1}<0, \alpha>0, a>0$ and $b>0$ are given numbers, and that $\varphi: \mathbb{R} \rightarrow(0, \infty)$ is a continuously differentiable function with $\varphi(\alpha)<\varphi(0), \varphi^{\prime}(0)>0$ and $\varphi^{\prime}(\alpha)>0$. Suppose that the i-v characteristics $F_{1}$ of SCR and $F_{2}$ of the Zener diode are defined by

$$
F_{1}(z)=\left\{\begin{array}{ll}
{\left[V_{1}, \varphi(0)\right],} & z=0, \\
a z+V_{1}, & z<0, \\
\varphi(z), & z \in[0, \alpha], \\
a(z-\alpha)+\varphi(\alpha), & z>\alpha
\end{array} \quad F_{2}(z)= \begin{cases}{[-V, V],} & z=0 \\
b z-V, & z<0 \\
b z+V, & z>0\end{cases}\right.
$$

Using the Kirchhoff's current and voltage laws, we have

$$
\left\{\begin{array}{l}
i=i_{1}+i_{2} \\
-u+R\left(i_{1}+i_{2}\right)+V_{s c r}+E_{1}=0 \\
-u+R\left(i_{1}+i_{2}\right)+V_{z d}+E_{2}=0 .
\end{array}\right.
$$

By setting $p_{1}=u-E_{1}, p_{2}=u-E_{2}, z_{1}=i_{1}$ and $z_{2}=i_{2}$, we obtain that

$$
p_{1} \in R\left(z_{1}+z_{2}\right)+F_{1}\left(z_{1}\right) \quad \text { and } \quad p_{2} \in R\left(z_{1}+z_{2}\right)+F_{2}\left(z_{2}\right)
$$

which is in the form of (1) with $m=n=2, B=C=I_{2}$, and $f(z)=A z, z \in \mathbb{R}^{2}$, where $A=\left(\begin{array}{ll}R & R \\ R & R\end{array}\right)$. Given $\bar{p}=\left(\bar{p}_{1}, \bar{p}_{2}\right) \in \mathbb{R}^{2}$, denote by $\bar{z}=\left(\bar{z}_{1}, \bar{z}_{2}\right)^{T}$ the corresponding solution to (1). In view of Remark 1 , the necessary and sufficient condition for the Aubin/Lipschitz-like property of $S$ at the reference point is

$$
\left.\begin{array}{l}
\left(R\left(\xi_{1}+\xi_{2}\right), \xi_{1}\right) \in-N\left(\left(\bar{z}_{1}, \bar{p}_{1}-R\left(\bar{z}_{1}+\bar{z}_{2}\right)\right) ; \operatorname{gph} F_{1}\right) \\
\left(R\left(\xi_{1}+\xi_{2}\right), \xi_{2}\right) \in-N\left(\left(\bar{z}_{2}, \bar{p}_{2}-R\left(\bar{z}_{1}+\bar{z}_{2}\right)\right) ; \operatorname{gph} F_{2}\right)
\end{array}\right\} \Longrightarrow \xi_{1}=\xi_{2}=0,
$$


where the first normal cone was computed in the Example 1-Silicon controlled rectifier and the latter one is given by $N\left((x, y)\right.$; gph $\left.F_{2}\right)$

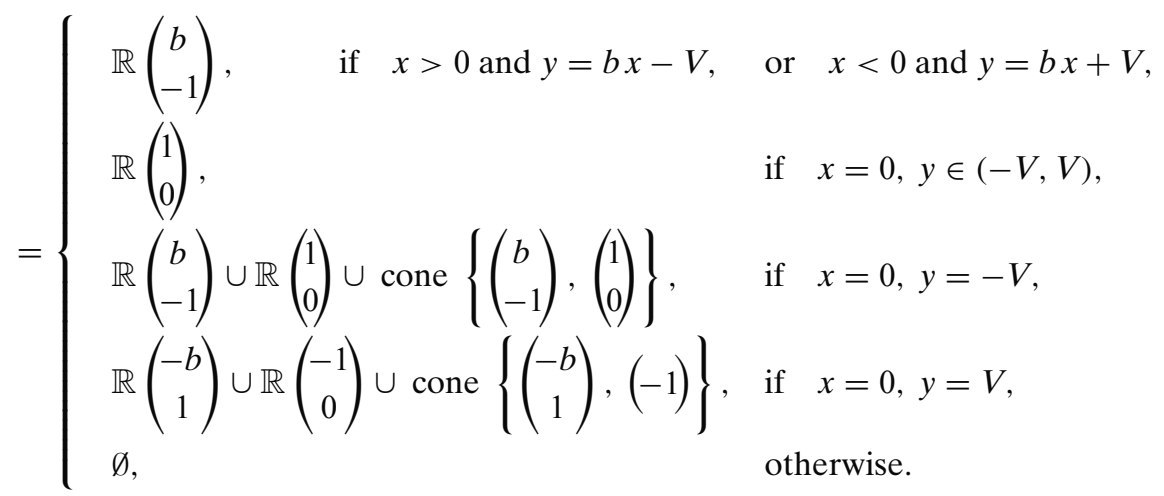

Multiplying the inclusions in (12) by the matrices $M_{1}:=\left(\begin{array}{cc}0 & 1 \\ 1 / R & -1\end{array}\right)$ and $M_{2}:=$ $\left(\begin{array}{cc}1 / R & -1 \\ 0 & 1\end{array}\right)$, respectively, one gets the following equivalent condition

$$
M_{1}\left(N\left(\left(\bar{z}_{1}, \bar{v}_{1}\right) ; \text { gph } F_{1}\right)\right) \bigcap M_{2}\left(N\left(\left(\bar{z}_{2}, \bar{v}_{2}\right) ; \text { gph } F_{2}\right)\right)=0_{\mathbb{R}^{2}} .
$$

Now, one infers that $M_{1}\left(N\left((x, y)\right.\right.$; gph $\left.\left.F_{1}\right)\right)$ is given by

$$
\begin{aligned}
& \mathbb{R}\left(\begin{array}{c}
-1 \\
\frac{a}{R}+1
\end{array}\right), \quad \text { if } \quad x<0 \text { and } y=a x+V_{1}, \quad \text { or } \quad x>\alpha \text { and } \quad y=a(x-\alpha)+\varphi(\alpha), \\
& \mathbb{R}\left(\begin{array}{l}
0 \\
\frac{1}{R}
\end{array}\right) \\
& \mathbb{R}\left(\begin{array}{c}
-1 \\
\frac{\varphi^{\prime}(x)}{R}+1
\end{array}\right) \\
& \left\{\mathbb{R}\left(\begin{array}{c}
-1 \\
\frac{a}{R}+1
\end{array}\right) \cup \mathbb{R}\left(\begin{array}{c}
0 \\
\frac{1}{R}
\end{array}\right) \cup \text { cone }\left\{\left(\begin{array}{c}
-1 \\
\frac{a}{R}+1
\end{array}\right),\left(\begin{array}{c}
0 \\
\frac{1}{R}
\end{array}\right)\right\}, \quad \text { if } \quad x=0, y=V_{1},\right. \\
& \mathbb{R}\left(\begin{array}{c}
1 \\
\frac{-\varphi^{\prime}(0)}{R}-1
\end{array}\right) \cup \mathbb{R}\left(\begin{array}{c}
0 \\
\frac{-1}{R}
\end{array}\right) \cup \text { cone }\left\{\left(\begin{array}{c}
1 \\
\frac{-\varphi^{\prime}(0)}{R}-1
\end{array}\right),\left(\begin{array}{c}
0 \\
\frac{-1}{R}
\end{array}\right)\right\}, \quad \text { if } \quad x=0, y=\varphi(0),
\end{aligned}
$$

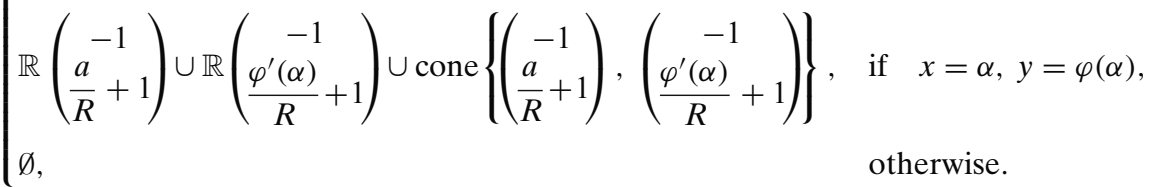


And $M_{2}\left(N\left((x, y) ; \operatorname{gph} F_{2}\right)\right)$ is given by

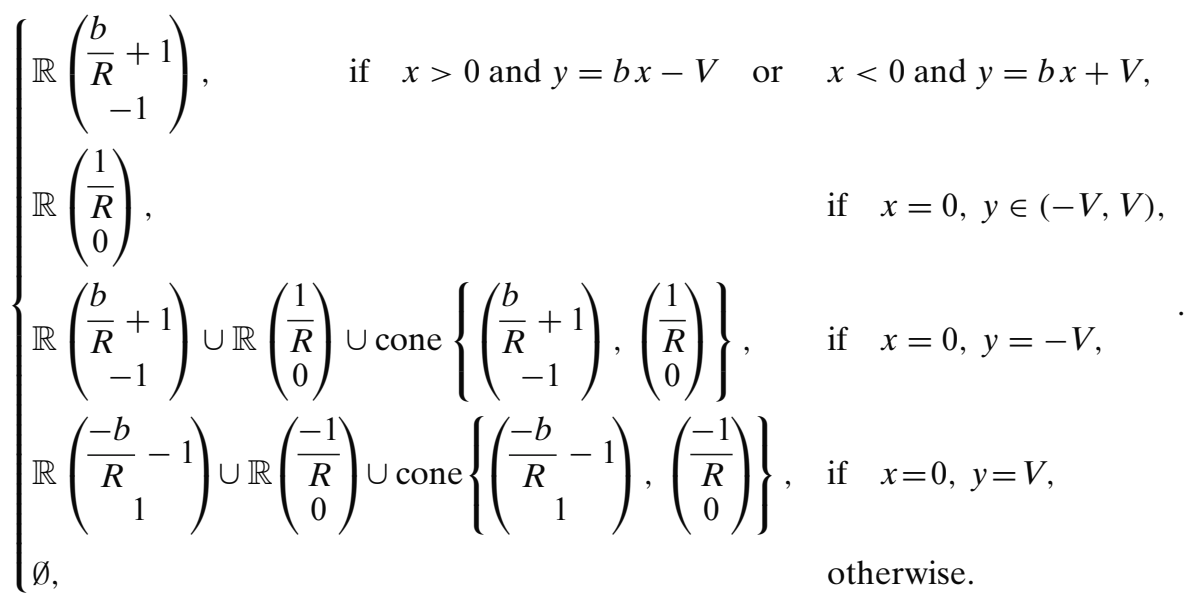

Again, in view of Remark 1, the necessary and sufficient condition for the isolated calmness property of $S$ at the reference point is

$$
\left.\begin{array}{l}
\left(b_{1},-R\left(b_{1}+b_{2}\right)\right) \in T\left(\left(\bar{z}_{1}, \bar{p}_{1}-R\left(\bar{z}_{1}+\bar{z}_{2}\right)\right) ; \operatorname{gph} F_{1}\right) \\
\left(b_{2},-R\left(b_{1}+b_{2}\right)\right) \in T\left(\left(\bar{z}_{2}, \bar{p}_{2}-R\left(\bar{z}_{1}+\bar{z}_{2}\right)\right) ; \operatorname{gph} F_{2}\right)
\end{array}\right\} \Longrightarrow b_{1}=b_{2}=0
$$

where the first tangent cone was computed in the Example 1 -Silicon controlled rectifier and $T\left((x, y)\right.$; gph $\left.F_{2}\right)$ is given by

$$
\begin{cases}\mathbb{R}\left(\begin{array}{l}
1 \\
b
\end{array}\right), & \text { if } x<0 \text { and } y=b x-V>0 \text { and } y=b x+V, \\
\mathbb{R}\left(\begin{array}{l}
0 \\
1
\end{array}\right), & \text { if } x=0, y \in(-V, V), \\
\mathbb{R}_{+}\left(\begin{array}{l}
-1 \\
-b
\end{array}\right) \cup \mathbb{R}_{+}\left(\begin{array}{l}
0 \\
1
\end{array}\right), & \text { if } x=0, y=-V, \\
\mathbb{R}_{+}\left(\begin{array}{l}
1 \\
b
\end{array}\right) \cup \mathbb{R}_{+}\left(\begin{array}{c}
0 \\
-1
\end{array}\right), & \text { if } x=0, y=V, \\
\emptyset, & \text { otherwise. }\end{cases}
$$

Multiplying the inclusions in (13) by the matrices $M_{3}:=\left(\begin{array}{cc}1 & 0 \\ -1 & -1 / R\end{array}\right)$ and $M_{4}:=$ $\left(\begin{array}{cc}-1 & -1 / R \\ 1 & 0\end{array}\right)$, respectively, one gets the following equivalent condition

$$
M_{3}\left(T\left(\left(\bar{z}_{1}, \bar{v}_{1}\right) ; \operatorname{gph} F_{1}\right)\right) \bigcap M_{4}\left(T\left(\left(\bar{z}_{2}, \bar{v}_{2}\right) ; \operatorname{gph} F_{2}\right)\right)=0_{\mathbb{R}^{2}}
$$


Hence, $M_{3}\left(T\left((x, y) ; \operatorname{gph} F_{1}\right)\right)$ is given by

$$
\begin{cases}\mathbb{R}\left(\begin{array}{c}
1 \\
-a / R-1
\end{array}\right), \quad \text { if } x<0, y=a x+V_{1} & \text { or } x>\alpha, y=a(x-\alpha)+\varphi(\alpha), \\
\mathbb{R}\left(\begin{array}{c}
0 \\
-1 / R
\end{array}\right), & \text { if } x=0, y \in\left(V_{1}, \varphi(0)\right), \\
\mathbb{R}\left(\begin{array}{c}
1 \\
-\varphi^{\prime}(x) / R-1
\end{array}\right), & \text { if } x \in(0, \alpha), y=\varphi(x), \\
\mathbb{R}_{+}\left(\begin{array}{c}
-1 \\
a / R+1
\end{array}\right) \cup \mathbb{R}_{+}\left(\begin{array}{c}
0 \\
-1 / R
\end{array}\right), & \text { if } x=0, y=V_{1}, \\
\mathbb{R}_{+}\left(\begin{array}{c}
1 \\
-\varphi^{\prime}(0) / R-1
\end{array}\right) \cup \mathbb{R}_{+}\left(\begin{array}{c}
0 \\
1 / R
\end{array}\right), & \text { if } x=0, y=\varphi(0), \\
\mathbb{R}_{+}\left(\begin{array}{c}
-1 \\
\varphi^{\prime}(\alpha) / R+1
\end{array}\right) \cup \mathbb{R}_{+}\left(\begin{array}{c}
1 \\
-a / R-1
\end{array}\right), & \text { if } x=\alpha, y=\varphi(\alpha), \\
\emptyset, & \text { otherwise. }\end{cases}
$$

And $M_{4}\left(T\left((x, y)\right.\right.$; gph $\left.\left.F_{2}\right)\right)$ is given by

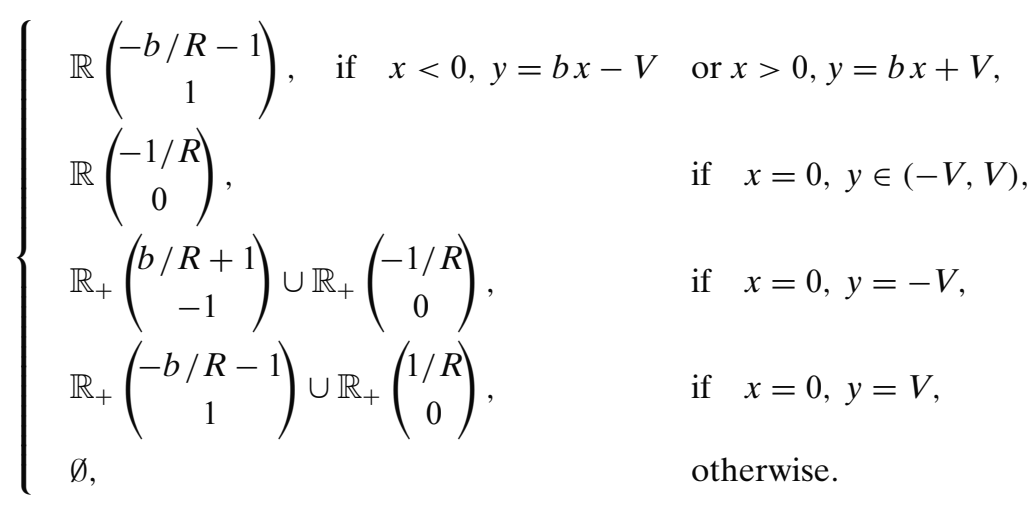

Hence, the properties of the solution map depend on the parameters of a particular circuit. For the sake of completeness, let us illustrate this on the isolated calmness property. For example, assume that $R=10, V_{1}=1, a=0.5, \alpha=1.3, \varphi(z)=24 z^{3}-$ $46 z^{2}+19 z+4, V=5$ and $b=1$, as in the simulation (see Fig. 9). For any $\left(x_{1}, y_{1}\right) \in$ gph $F_{1}$ with $x_{1} \notin(0,1.3)$ and any $\left(x_{2}, y_{2}\right) \in$ gph $F_{2}$ one has

$$
M_{3}\left(T\left(\left(x_{1}, y_{1}\right) ; \operatorname{gph} F_{1}\right)\right) \subset \mathbb{R}\left\{\left(\begin{array}{c}
-1 \\
1.05
\end{array}\right),\left(\begin{array}{c}
-1 \\
2.9
\end{array}\right),\left(\begin{array}{c}
-1 \\
3.08
\end{array}\right),\left(\begin{array}{l}
0 \\
1
\end{array}\right)\right\}
$$

and

$$
M_{4}\left(T\left(\left(x_{2}, y_{2}\right) ; \operatorname{gph} F_{2}\right)\right) \subset \mathbb{R}\left\{\left(\begin{array}{c}
-1.1 \\
1
\end{array}\right),\left(\begin{array}{l}
1 \\
0
\end{array}\right)\right\}=\mathbb{R}\left\{\left(\begin{array}{c}
-1 \\
1 / 1.1
\end{array}\right),\left(\begin{array}{l}
1 \\
0
\end{array}\right)\right\}
$$



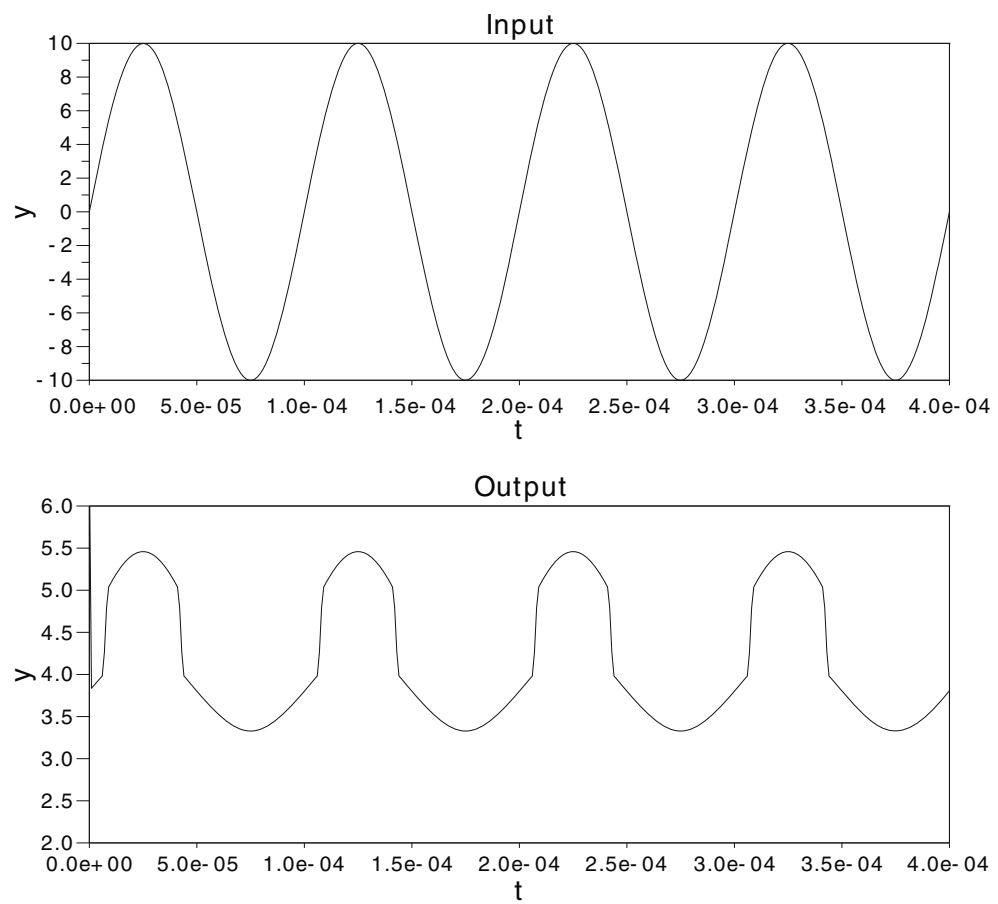

Fig. 9 Voltage of the input source and the output voltage between the nodes $\mathrm{G}$ and $\mathrm{H}$ in Fig. 7 $\left(R=10, V_{1}=1, a=0.5, \alpha=1.3, \varphi(z)=24 z^{3}-46 z^{2}+19 z+4, V=5, b=1, \varepsilon=0.01\right)$

If $x_{1} \in(0,1.3)$ then we loose the isolated calmness property provided it is a solution to the equation

$$
\left(72 x^{2}-92 x+19\right) / 10+1=1 / 1.1,
$$

which happens precisely for two points lying in the interval $(0,1.3)$. Therefore, $S$ has the isolated calmness property at $(\bar{p}, \bar{z})$ if and only if $\bar{z}_{1}$ does not solve the above mentioned quadratic equation.

Example 3 (Transistor) Consider the circuit in Fig. 10 involving load resistances $R_{B}>0$ and $R_{L}>0$, two input-signal sources $u_{1}$ and $u_{2}$, and a P-N-P transistor (see Fig. 11) having three terminals labeled emitter, base and collector. Its behaviour can be described by the Ebers-Moll model [7, p. 409] involving two diodes placed back to back and two dependent current-controlled sources $\alpha_{I} I^{\prime}$ and $\alpha_{N} I$ shunting the diodes. Here $\alpha_{N} \in[0,1)$ is known as the current gain in normal operation and $\alpha_{I} \in$ $(0,1]$ is known as the inverted common-base gain current. Therefore $i_{E}=I-\alpha_{I} I^{\prime}$ and $i_{C}=I^{\prime}-\alpha_{N} I$. This means that

$$
\left(\begin{array}{l}
i_{E} \\
i_{C}
\end{array}\right)=\left(\begin{array}{cc}
1 & -\alpha_{I} \\
-\alpha_{N} & 1
\end{array}\right)\left(\begin{array}{l}
I \\
I^{\prime}
\end{array}\right) .
$$

Kirchhoff's laws also reveal that $i_{B}=-\left(i_{E}+i_{C}\right)$, so $R_{B}\left(-i_{C}-i_{E}\right)+u_{1}-V_{E}=0$ and $0=V_{C}+u_{2}+R_{L} i_{C}-V_{E}=V_{C}+u_{2}+R_{L} i_{C}+R_{B}\left(i_{C}+i_{E}\right)-u_{1}$. Given $V_{E 1}<$ 
Fig. 10 The circuit considered in Example 3

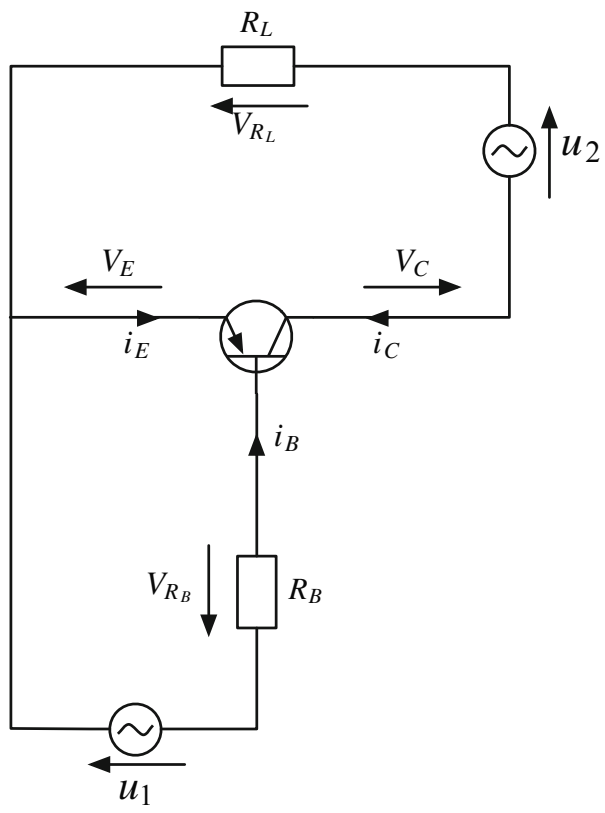

$0<V_{E 2}$ and $V_{C 1}<0<V_{C 2}$, assume that the characteristics of the diodes involved in Ebers-Moll model are defined by

$$
F_{1}(x):=\left\{\begin{array}{ll}
{\left[V_{E 1}, V_{E 2}\right],} & x=0, \\
V_{E 1}, & x<0, \\
V_{E 2}, & x>0,
\end{array} \quad \text { and } \quad F_{2}(x):= \begin{cases}{\left[V_{C 1}, V_{C 2}\right],} & x=0, \\
V_{C 1}, & x<0, \\
V_{C 2}, & x>0,\end{cases}\right.
$$

one has $V_{E} \in F_{1}(I)$ and $V_{C} \in F_{2}\left(I^{\prime}\right)$. To sum up, we obtained that

$$
\left(\begin{array}{c}
u_{1} \\
u_{1}-u_{2}
\end{array}\right) \in \underbrace{\left(\begin{array}{cc}
R_{B} & R_{B} \\
R_{B} & R_{B}+R_{L}
\end{array}\right)\left(\begin{array}{cc}
1 & -\alpha_{I} \\
-\alpha_{N} & 1
\end{array}\right)}_{=: A}\left(\begin{array}{l}
I \\
I^{\prime}
\end{array}\right)+\left(\begin{array}{l}
F_{1}(I) \\
F_{2}\left(I^{\prime}\right)
\end{array}\right) .
$$
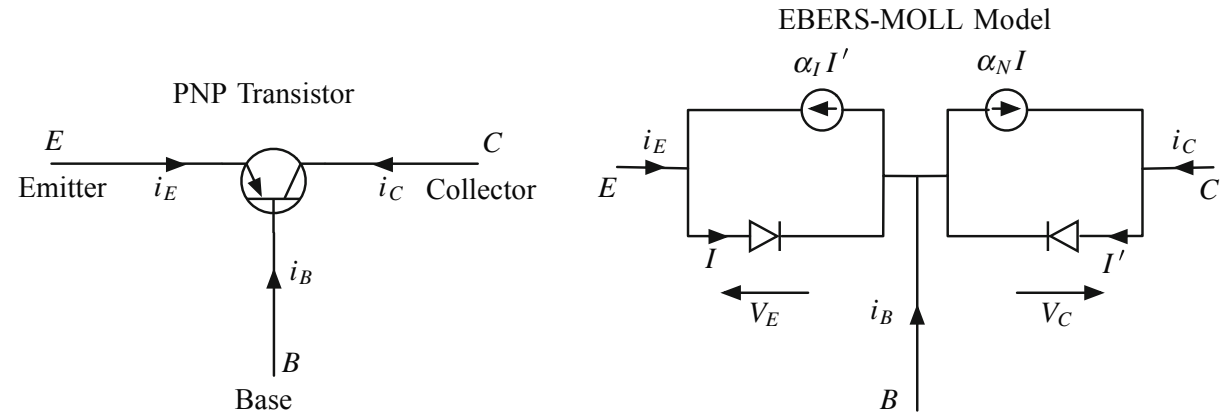

Fig. 11 The P-N-P transistor and its Ebers-Moll model 
So we arrived at (1) with $n=m=2, B=C=I_{2}, p:=\left(u_{1}, u_{1}-u_{2}\right)^{T}, z:=\left(I, I^{\prime}\right)^{T}$, $f(z):=A z$ and $F(z)=\left(F_{1}\left(z_{1}\right), F_{2}\left(z_{2}\right)\right)^{T}$.

Fix $\bar{p} \in \mathbb{R}^{2}$ and let $\bar{z} \in \mathbb{R}^{2}$ be the corresponding unique solution to (1) which exists due to [3, Theorem 2.1]. Note that

$$
A^{T}=\left(\begin{array}{cc}
\left(1-\alpha_{N}\right) R_{B} & \left(1-\alpha_{N}\right) R_{B}-\alpha_{N} R_{L} \\
\left(1-\alpha_{I}\right) R_{B} & \left(1-\alpha_{I}\right) R_{B}+R_{L}
\end{array}\right) .
$$

The principal minors of $A^{T}$ equal to

$$
\left(1-\alpha_{N}\right) R_{B}>0, \quad\left(1-\alpha_{I}\right) R_{B}+R_{L}>0, \quad \text { and } \quad \operatorname{det} A^{T}=\left(1-\alpha_{I} \alpha_{N}\right) R_{L} R_{B}>0 .
$$

Therefore, $A^{T}$ is a P-matrix. As both $F_{1}$ and $F_{2}$ are maximal monotone, Corollary 1 says that $S$ has the Aubin/Lipschitz-like property at the reference point. The uniqueness of the solution guarantees the isolated calmness property of $S$. The simulation can be seen in Fig. 12.
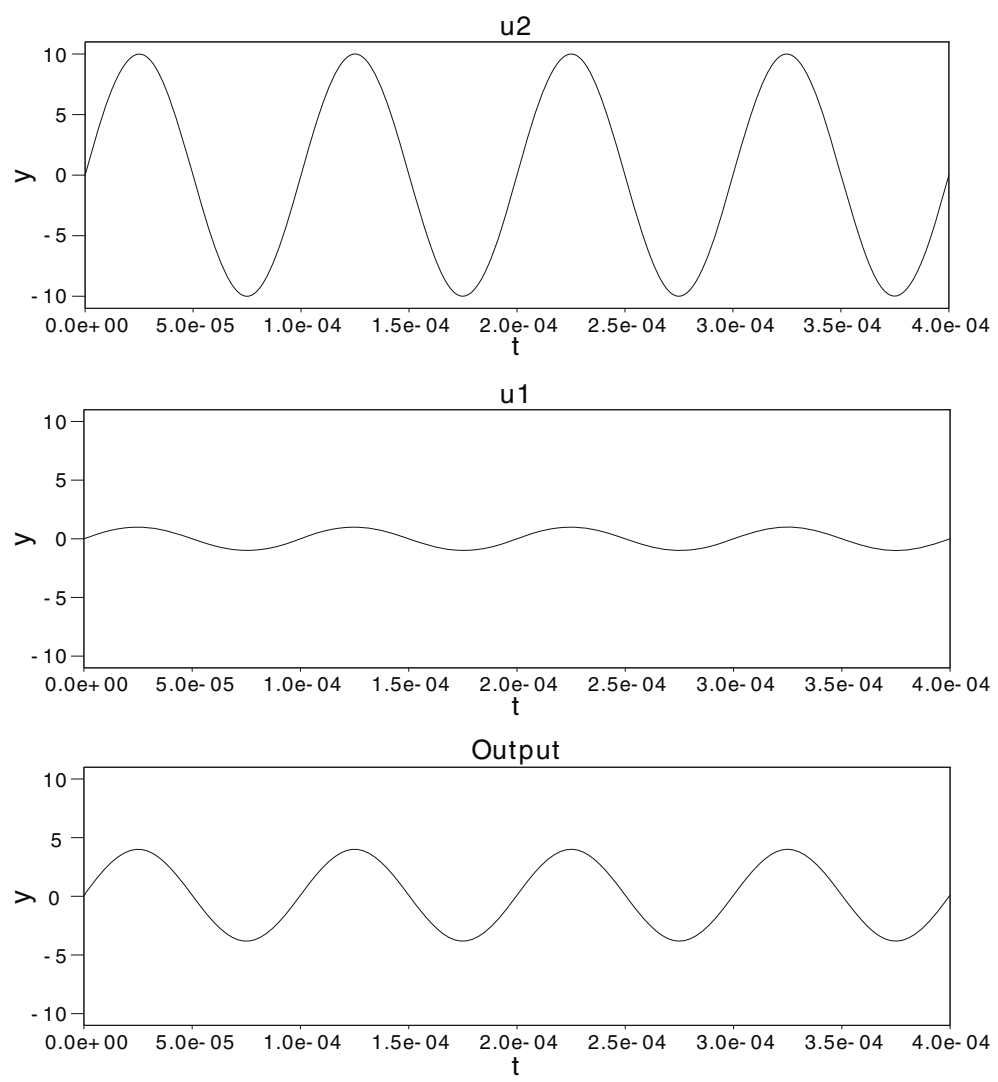

Fig. 12 Input and Output voltage $\left(R_{L}=3000, R_{B}=30000, \alpha_{I}=0.7, \alpha_{N}=0.1, V_{E 1}=-15, V_{E 2}=\right.$ $1, V_{C 1}=-15, V_{C 2}=1$, and $\left.\varepsilon=0.01\right)$. The output voltage is measured between the Emitter and the Collector 


\section{Isolated Calmness Without Injectivity Assumption}

We have assumed that $m \leq n$. However, one can have $m>n$ in some applications. In such a case, one cannot expect the assumptions $(A 1)$ and $(A 4)$ to hold. From now on, $m, n \in \mathbb{N}$ are not related to each other by an inequality. Let us define a set-valued mapping $F_{C}: \mathbb{R}^{m} \rightrightarrows \mathbb{R}^{m}$ by $F_{C}(u)=F(u)$ if $u=C z$ for some $z \in \mathbb{R}^{n}$ and $F_{C}(u)=\emptyset$ otherwise. We assume the following (instead of $(A 1))$ :

( $\widetilde{A} 1)$ Suppose that there is $\bar{v} \in F(C \bar{z})$ such that

$$
\bar{p}=f(\bar{z})+B \bar{v} \quad \text { and } \overline{\bigcup_{t>0} \frac{\operatorname{rge} F_{C}-\bar{v}}{t}} \bigcap \operatorname{ker} B=\left\{0_{\mathbb{R}^{m}}\right\} .
$$

Of course, if $\bar{v}$ is an interior point of $\operatorname{rge} F_{C}$ then $(\widetilde{A} 1)$ reduces to $(A 1)$. Let us start with two geometrical lemmas.

Lemma 1 Let $E \in \mathbb{R}^{k \times d}$ be any matrix and $\Gamma$ be a subset of rge $E$. Put $\Xi=E^{-1}(\Gamma)$. Then

$$
T(\bar{y} ; \Xi)=\left\{w \in \mathbb{R}^{d}: E w \in T(E \bar{y}, \Gamma)\right\} \quad \text { whenever } \quad \bar{y} \in \Xi
$$

Proof This was shown in the first part of the proof of [Adly, S., Cibulka, R.: Quantitative stability of a generalized equation. Application to non-regular electrical circuits. to appear in J. Optim. Theory Appl., Lemma 6].

Lemma 2 Let $G \in \mathbb{R}^{l \times d}$, let $\Xi \subset \mathbb{R}^{d}$, and set $\Lambda=G(\Xi)$. Suppose that $\bar{x} \in \Lambda$ and $\bar{y} \in \Xi$ are such that

$$
G(\bar{y})=\bar{x} \quad \text { and } \overline{\bigcup_{t>0} \frac{\Xi-\bar{y}}{t}} \bigcap \operatorname{ker} G=\left\{0_{\mathbb{R}^{d}}\right\} .
$$

Then

$$
T(\bar{x} ; \Lambda)=G(T(\bar{y} ; \Xi))
$$

Proof To prove that $G(T(\bar{y} ; \Xi)) \subset T(\bar{x} ; \Lambda)$, pick any $w \in G(T(\bar{y} ; \Xi))$. Find $v \in$ $T(\bar{y} ; \Xi)$ with $G(v)=w$. Thus there is $\left(t^{n}\right)_{n \in \mathbb{N}}$ in $(0, \infty)$ converging to 0 and $\left(v^{n}\right)_{n \in \mathbb{N}}$ in $\mathbb{R}^{d}$ converging to $v$ such that $\bar{y}+t^{n} v^{n} \in \Xi$ whenever $n \in \mathbb{N}$. For each $n \in \mathbb{N}$, put $w^{n}:=G\left(v^{n}\right)$. Clearly, $\left(w^{n}\right)_{n \in \mathbb{N}}$ converges to $w$. Moreover,

$$
\bar{x}+t^{n} w^{n}=G\left(\bar{y}+t^{n} v^{n}\right) \in G(\Xi)=\Lambda \quad \text { whenever } \quad n \in \mathbb{N} .
$$

So $w \in T(\bar{x} ; \Lambda)$. To see the opposite inclusion, pick any $w \in T(\bar{x} ; \Lambda)$. Find $\left(t^{n}\right)_{n \in \mathbb{N}}$ in $(0, \infty)$ converging to 0 and $\left(w^{n}\right)_{n \in \mathbb{N}}$ in $\mathbb{R}^{l}$ converging to $w$ such that

$$
G(\bar{y})+t^{n} w^{n} \in G(\Xi) \text { for each } n \in \mathbb{N} .
$$

For each $n \in \mathbb{N}$, find $v^{n} \in \Xi$ such that $w^{n}=G\left(\left(v^{n}-\bar{y}\right) / t^{n}\right)$, and put $u_{n}=\left(v^{n}-\bar{y}\right) / t^{n}$. We claim that $\left(u^{n}\right)_{n \in \mathbb{N}}$ is bounded. Suppose on the contrary that this is not the case. Passing to a subsequence if necessary, we may assume that $\left(u^{n} /\left\|u^{n}\right\|\right)_{n \in \mathbb{N}}$ converges, to $\bar{u} \in S_{\mathbb{R}^{d}}$, say. For each $n \in \mathbb{N}$, one has that

$$
\frac{u^{n}}{\left\|u^{n}\right\|}=\frac{v^{n}-\bar{y}}{\left\|v^{n}-\bar{y}\right\|} \quad \text { and } \quad \frac{w^{n}}{\left\|u^{n}\right\|}=G\left(\frac{u^{n}}{\left\|u^{n}\right\|}\right) .
$$


Passing to the limit for $n \rightarrow \infty$ one gets, that $\bar{u} \in \overline{\bigcup_{t>0} \frac{\Xi-\bar{y}}{t}}$ and $0=G(\bar{u})$, a contradiction since $\bar{u} \neq 0$. Having the claim in hand, one may assume without a loss of generality that $\left(u^{n}\right)_{n \in \mathbb{N}}$ converges to some $u \in \mathbb{R}^{d}$. For each $n \in \mathbb{N}$, we have $\bar{y}+t^{n} u^{n}=$ $v^{n} \in \Xi$, therefore $u \in T(\bar{y} ; \Xi)$. Moreover, $w=G(u)$. The proof is finished.

The above statement is false without an additional assumption on a relation between the set and the kernel of the matrix $G$.

Example 4 Let $l=1, d=2, \quad G=\left(\begin{array}{ll}1 & 0\end{array}\right), \quad \Xi=\operatorname{gph} \partial|\cdot|, \quad \bar{y}=(0,0)^{T}$ and $\bar{x}=0$. Then $G(\bar{y})=\bar{x}, \Lambda=G(\Xi)=\mathbb{R}$ and $T(\bar{y}, \Xi)=\{0\} \times \mathbb{R}$. So $\{0\}=G(T(\bar{y}, \Xi)) \neq$ $T(\bar{x}, \Lambda)=\mathbb{R}$.

Given $H: \mathbb{R}^{d} \rightrightarrows \mathbb{R}^{l}$, the contingent (graphical) derivative of $H$ at $(\bar{x}, \bar{y}) \in \operatorname{gph} H$ is the mapping $D H(\bar{x}, \bar{y}): \mathbb{R}^{d} \rightrightarrows \mathbb{R}^{l}$ defined by

$$
D H(\bar{x}, \bar{y})(u)=\left\{v \in \mathbb{R}^{l}:(u, v) \in T((\bar{x}, \bar{y}) ; \operatorname{gph} H)\right\}, \quad u \in \mathbb{R}^{d} .
$$

Proposition 1 Assume that $(\widetilde{A} 1)$ and $(A 2)$ hold true. Then

$$
D \Phi(\bar{z}, \bar{p})(b)=\nabla f(\bar{z}) b+B D F_{C}(C \bar{z}, \bar{v})(C b) \quad \text { whenever } \quad b \in \mathbb{R}^{n} .
$$

Proof Fix any $b \in \mathbb{R}^{n}$. By [2, Proposition 4A.2], we have

$$
D \Phi(\bar{z}, \bar{p})(b)=\nabla f(\bar{z}) b+D Q(\bar{z}, \bar{p}-f(\bar{z}))(b) .
$$

Define $H: \mathbb{R}^{n} \rightrightarrows \mathbb{R}^{m}$ by $H(z):=F(C z), z \in \mathbb{R}^{n}$. Then

$$
\operatorname{gph} Q=G(\operatorname{gph} H) \quad \text { with } \quad G:=\left(\begin{array}{cc}
I_{n} & 0 \\
0 & B
\end{array}\right)
$$

Then $\operatorname{ker} G=\left\{0_{\mathbb{R}^{n}}\right\} \times \operatorname{ker} B$ and gph $H \subset \mathbb{R}_{n} \times \operatorname{rge} F_{C}$. Therefore

$$
\overline{\bigcup_{t>0} \frac{\operatorname{gph} H-(\bar{z}, \bar{v})}{t}} \bigcap \operatorname{ker} G \subset\left\{0_{\mathbb{R}^{n}}\right\} \times\left(\overline{\bigcup_{t>0} \frac{\operatorname{rge} F_{C}-\bar{v}}{t}} \bigcap \operatorname{ker} B\right)=\left\{0_{\mathbb{R}^{n+m}}\right\} .
$$

Lemma $2($ with $l:=2 n, d:=n+m, \Xi:=\operatorname{gph} H, \bar{x}=(\bar{z}, \bar{p}-f(\bar{z}))$, and $\bar{y}=(\bar{z}, \bar{v}))$ reveals that

$$
T((\bar{z}, \bar{p}-f(\bar{z})) ; \operatorname{gph} Q)=\left\{\left(\begin{array}{c}
b \\
B c
\end{array}\right):\left(\begin{array}{l}
b \\
c
\end{array}\right) \in T((\bar{z}, \bar{v}) ; \text { gph } H)\right\} .
$$

This means that $D Q(\bar{z}, \bar{p}-f(\bar{z}))(b)=B D H(\bar{z}, \bar{v})(b)$. Moreover,

$$
\text { gph } H=\left\{\left(\begin{array}{l}
z \\
v
\end{array}\right) \in \mathbb{R}^{n} \times \mathbb{R}^{m}: E\left(\begin{array}{l}
z \\
v
\end{array}\right) \in \text { gph } F_{C}\right\} \quad \text { with } \quad E:=\left(\begin{array}{cc}
C & 0 \\
0 & I_{m}
\end{array}\right) \text {. }
$$


Lemma 1 (with $k:=2 m, d:=n+m, \Gamma:=\operatorname{gph} F_{C}$, and $\left.\bar{y}=(\bar{z}, \bar{v})\right)$ reveals that

$$
T((\bar{z}, \bar{v}) ; \operatorname{gph} H)=\left\{\left(\begin{array}{l}
b \\
c
\end{array}\right) \in \mathbb{R}^{n} \times \mathbb{R}^{m}: E\left(\begin{array}{l}
b \\
c
\end{array}\right) \in T\left((C \bar{z}, \bar{v}) ; \operatorname{gph} F_{C}\right)\right\} .
$$

Hence $D H(\bar{z}, \bar{v})(b)=D F_{C}(C \bar{z}, \bar{v})(C b)$. Combining the above facts, the statement follows.

Theorem 3 Suppose that ( $\widetilde{A 1}),(A 2)$ and $(A 3)$ hold true. Then $S$ has the isolated calmness property at $(\bar{p}, \bar{z})$ if and only if

$$
0 \in \nabla f(\bar{z}) b+B D F_{C}(C \bar{z}, \bar{v})(C b) \quad \Rightarrow \quad b=0_{\mathbb{R}^{n}} .
$$

Proof Note that $x \in D \Phi(\bar{z}, \bar{p})(y)$ if and only if $y \in D S(\bar{p}, \bar{z})(x)$. Combine [2, Corollary 4C.2 and Theorem 4C.1] and Proposition 1 to conclude the proof.

To use the above statement, one has to know the range of the matrix $C$. Sometimes the following sufficient condition may be useful.

Corollary 2 Under the assumptions of Theorem 3, S has the isolated calmness property at $(\bar{p}, \bar{z})$ provided that $b=0_{\mathbb{R}^{n}}$ is the only point such that

$$
\nabla f(\bar{z}) b+B w=0 \quad \text { and } \quad(C b, w) \in T((C \bar{z}, \bar{v}) ; \text { gph } F) \quad \text { for some } \quad w \in \mathbb{R}^{m} .
$$

Proof Clearly, gph $F_{C} \subset \operatorname{gph} F$, so $T\left((C \bar{z}, \bar{v})\right.$; gph $\left.F_{C}\right) \subset T((C \bar{z}, \bar{v})$; gph $F)$. Take any $b \in \mathbb{R}^{n}$ such that $0 \in \nabla f(\bar{z}) b+B D F_{C}(C \bar{z}, \bar{v})(C b)$. Find $w \in \mathbb{R}^{m}$ such that $0=$ $\nabla f(\bar{z}) b+B w$ and $(C b, w) \in T\left((C \bar{z}, \bar{v}) ; \operatorname{gph} F_{C}\right)$. Thus $b=0 \mathbb{R}_{\mathbb{R}^{n}}$.

Example 5 (Sampling gate) The circuit in Fig. 13 is a sampling gate involving a bridge of four diodes $D_{1}, D_{2}, D_{3}, D_{4}$ and symmetrically controlled by gate voltages $+V_{c}$ and $-V_{c}$ through the control resistors $R_{c}>0$. The input-signal is given by $V_{i}$ and the output signal is defined by the voltage $V_{o}$ through the load resistor $R_{L}>0$.

Fig. 13 Four-diode-bridge sampling gate

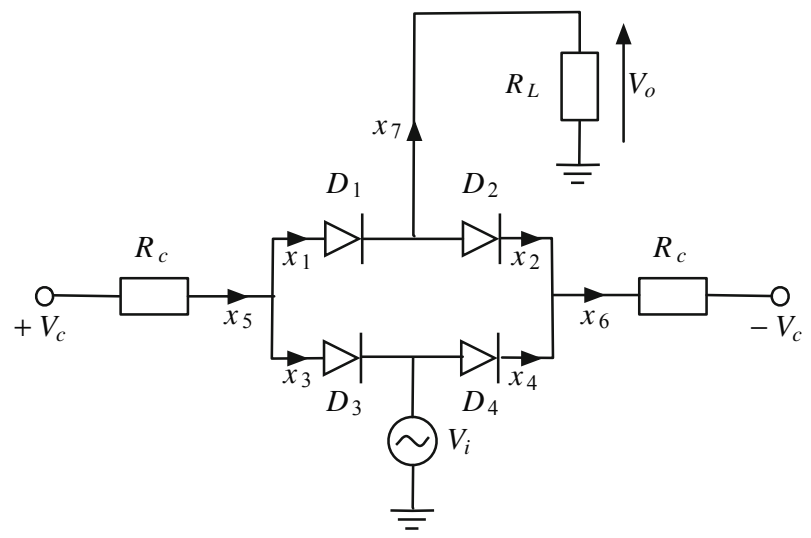


Given $j \in\{1,2,3,4\}$, denote by $V_{j}$ and $x_{j}$ the voltage and the current across the diode $D_{j}$, respectively. Moreover, let $x_{5}$ be the current through the left resistor $R_{c}, x_{6}$ be the current through the right resistor $R_{c}$ and $x_{7}$ be the current trough resistor $R_{L}$. Kirchhoff's laws yield that $V_{i}-V_{4}+V_{2}-R_{L} x_{7}=0,2 V_{c}-V_{3}-V_{4}-$ $R_{c} x_{6}-R_{c} x_{5}=0$, and $-V_{1}-V_{2}+V_{3}+V_{4}=0$. And also, that $x_{1}+x_{3}=x_{2}+x_{4}=$ $x_{5}=x_{6}$ and $x_{1}-x_{2}=x_{4}-x_{3}=x_{7}$. Put $z=\left(x_{7}, x_{6}, x_{1}\right)^{T}, v=\left(V_{1}, V_{2}, V_{3}, V_{4}\right)^{T}, u=$ $\left(V_{i}, 2 V_{c}\right)^{T}$,

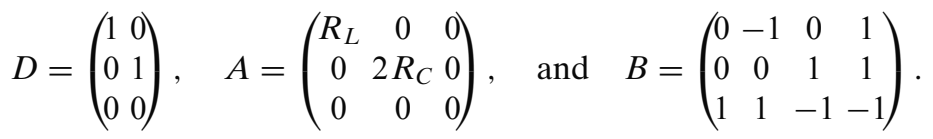

Hence, $D u=A z+B v$. Assume that all the diodes have the same characteristics given for $V_{D 1}<0<V_{D 2}$ by

$$
F_{D}(x):= \begin{cases}{\left[V_{D 1}, V_{D 2}\right],} & x=0, \\ V_{D 1}, & x<0 \\ V_{D 2}, & x>0 .\end{cases}
$$

Thus $V_{1} \in F_{D}\left(x_{1}\right), \quad V_{2} \in F_{D}\left(x_{2}\right)=F_{D}\left(x_{1}-x_{7}\right), \quad V_{3} \in F_{D}\left(x_{3}\right)=F_{D}\left(x_{6}-x_{1}\right)$, and $V_{4} \in F_{D}\left(x_{4}\right)=F_{D}\left(x_{7}+x_{6}-x_{1}\right)$. Putting $C=B^{T}$ and $F(y)=\left(F_{D}\left(y_{1}\right), F_{D}\left(y_{2}\right)\right.$,
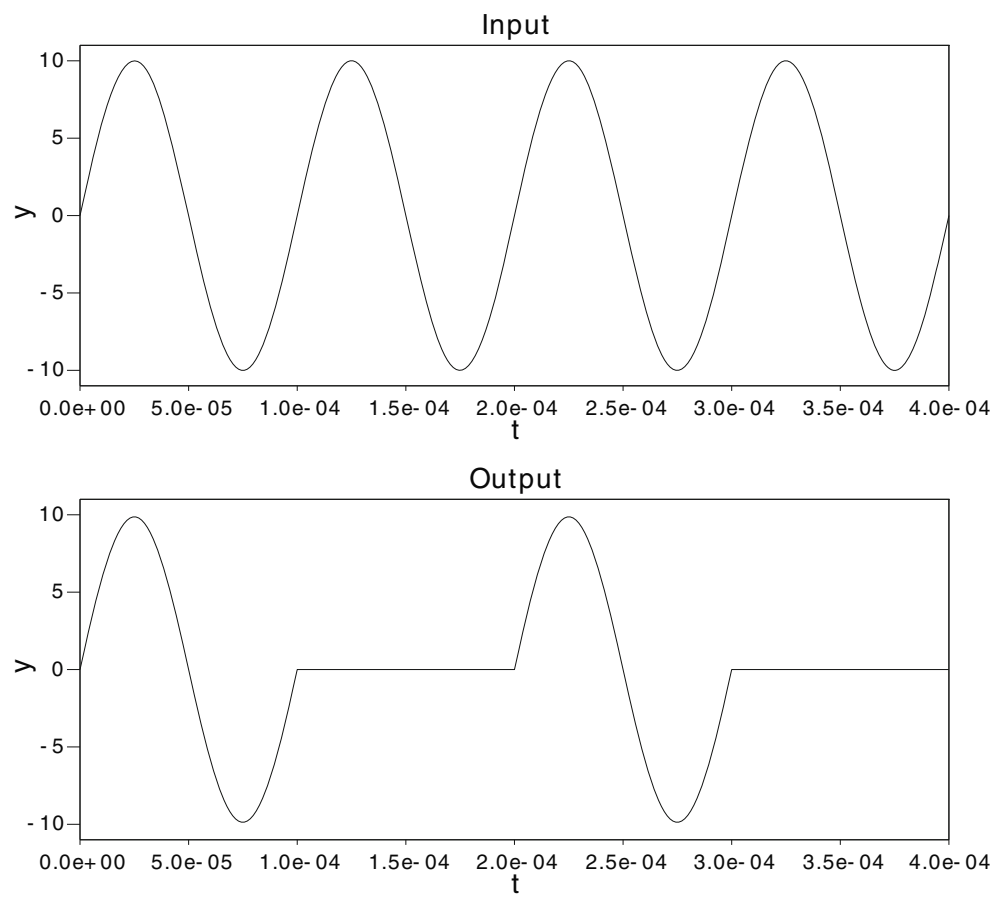

Fig. 14 Input voltage $V_{i}$ and the output voltage $V_{o}$ of the sampling gate $\left(R_{L}=1000, R_{C}=10\right.$, and $\left.V_{C}=11\right)$ 
$\left.F_{D}\left(y_{3}\right), F_{D}\left(y_{4}\right)\right)^{T}, y \in \mathbb{R}^{4}$, we have $v \in F(C z)$. So, we arrived at (1) with $n=3$, $m=4, p:=D u$, and $f(z):=A z$.

Let $\bar{p}=\left(0,2 V_{D 1}, 0\right)^{T}$ and $\bar{z}=(0,0,0)^{T}$. Put $\bar{v}=\left(V_{D 1}, V_{D 1}, V_{D 1}, V_{D 1}\right)^{T}$. Then

$$
(\bar{z}, \bar{p}) \in \operatorname{gph} \Phi, \quad \bar{p}=A \bar{z}+B \bar{v} \quad \text { and } \quad \bar{v} \in F(C \bar{z})
$$

Moreover rge $F_{C} \subset$ rge $F=\left[V_{D 1}, V_{D 2}\right]^{4}$. Therefore

$$
\overline{\bigcup_{t>0}^{\operatorname{rge} F_{C}-\bar{v}}} \bigcap^{t} \operatorname{ker} B \subset \mathbb{R}_{+}^{4} \bigcap \operatorname{ker} B=\mathbb{R}_{+}^{4} \bigcap \mathbb{R}\left\{(-1,1,-1,1)^{T}\right\}=\left\{0_{\mathbb{R}^{4}}\right\}
$$

Let $b \in \mathbb{R}^{3}$ be such that

$$
A b+B w=0 \quad \text { and } \quad(C b, w) \in T((0, \bar{v}) ; \operatorname{gph} F) \quad \text { for some } \quad w \in \mathbb{R}^{4}
$$

Clearly, $T((0, \bar{v}) ; \operatorname{gph} F)$ equals to

$$
\prod_{i=1}^{4} T\left(\left(0, V_{D 1}\right) ; \operatorname{gph} F_{D}\right)=\left[\left\{(x, y)^{T} \in \mathbb{R}^{2}: x \leq 0, y \geq 0, x y=0\right\}\right]^{4} .
$$

Hence, $0=(C b, w)=\left(B^{T} b, w\right)=(b, B w)=-(b, A b)=-R_{L} b_{1}^{2}-2 R_{C} b_{2}^{2}$. This reveals that $b_{1}=b_{2}=0$. So $C b=\left(b_{3}, b_{3},-b_{3},-b_{3}\right)^{T}$. All the coordinates of this vector have to be non-negative, so $b_{3}=0$. Applying Corollary 2 one concludes that $S$ has the isolated calmness property at the reference point. The simulation can be seen in Fig. 14.

\section{Conclusion}

We studied the quantitative stability of a solution mapping corresponding to several important circuits appearing in electronics and we provided their input-output simulation. We also showed that the assumption on injectivity (surjectivity) of the matrices involved in the model may be slightly weakened. It would be preferable to find a simpler and weaker qualification condition than $(\widetilde{A} 1)$ ensuring the validity of the corresponding calculus rules. This is out of the scope of the current manuscript and will be probably the aim of future work.

\section{References}

1. Adly, S., Outrata, J.V.: Qualitative stability of a class of non-monotone variational inclusions. Application in electronics, J. Convex Anal. 20, 1 (2013)

2. Dontchev, A.L., Rockafellar, R.T.: Implicit functions and solution mappings. A view from variational analysis. Springer Monographs in Mathematics. Springer, Dordrecht (2009)

3. Goeleven, D.: Existence and uniqueness for a linear mixed variational inequality arising in electrical circuits with transistors. J. Optim. Theory Appl. 138(3), 397-406 (2008) 
4. Mordukhovich, B.S.: Variational analysis and generalized differentiation. I. Basic theory. Grundlehren der Mathematischen Wissenschaften, vol. 330. Springer-Verlag, Berlin (2006)

5. Moreau, J.J., Panagiotopoulos, P.D.: Nonsmooth Mechanics and Applications. Springer-Verlag, Wien-New York (1988)

6. Rockafellar, R.T., Wets, R.J.-B.: Variational analysis. Grundlehren der Mathematischen Wissenschaften, vol. 317. Springer-Verlag, Berlin (1998)

7. Sedra, A.S., Smith, K.C.: Microelectronic Circuits, 5th edn. Oxford University Press, New York, Oxford (2004) 